\title{
DER TÄTER ALS ÜBERLEBENDER: DER KZ-ARZT IM KINO
}

\author{
OLIVER LUBRICH \\ Universität Bern \\ oliver.lubrich@germ.unibe.ch \\ NINA PETER \\ Universität Bern \\ nina.peter@germ.unibe.ch
}

\section{ABSTRACT}

Der Aufsatz gibt einen ersten Überblick über rund 30 Spielfilme, in denen die Figur des KZArztes (häufig gestaltet nach dem Vorbild Josef Mengeles) auftritt. Er untersucht deren populärkulturelle Faszination und erinnerungspolitische Bedeutungen. Die Filme werden thematisch und generisch in vier Gruppen analysiert: (1.) Der Täter am Tatort: Die Filme zeigen den Arzt im Konzentrationslager und sind so dem Genre des Holocaust-Films zuzuordnen. (2.) Der Täter als Überlebender: Die Filme spielen nach 1945 und thematisieren einerseits Flucht und Überleben des KZ-Arztes im Exil, andererseits seine Verfolgung und juristische Bewertung. (3.) Nachfahren: Die Filme rücken Figuren der Söhne- und TöchterGeneration und deren Verhältnis zum nationalsozialistischen Arzt in den Mittelpunkt. (4.) Action, Horror, Trash: KZ-Arzt-Darstellungen in populären Genres lösen die Figur aus ihrem historischen Kontext heraus, sie verselbständigt sich $\mathrm{zu}$ einer beliebig einsetzbaren Verkörperung des Bösen.

SCHLÜSSELWÖRTER: KZ-Arzt, Mengele, Holocaust, Nationalsozialismus, Film, kollektive Imagination

\section{THE SURVIVING PERPETRATOR: THE NAZI DOCTOR ON SCREEN}

ABSTRACT

This first comprehensive account of 30 movies that feature Nazi doctors (frequently based on Josef Mengele, doctor at Auschwitz) explores this figure's popular fascination and commemorative functions. The films are analysed in four thematic and generic groups: (1.) The perpetrator at the scene of the crime: Holocaust films show the Nazi doctor , at work' in the concentration camp. (2.) The perpetrator as a survivor: Many films deal with his escape and survival in exile as well as his persecution and legal reprobation. (3.) Descendants: These films focus on the generation of sons and daughters and their relation to the Nazi perpetrator. (4.) Action, Horror, Trash: Representations in popular genres isolate the Nazi doctor from his historical context, turning him into an arbitrary symbol of ,evil'.

KEYWORDS: Nazi doctor, Mengele, Holocaust, National socialism, Film, Collective imagination

\section{DER KZ-ARZT IM FILM}

In der Erinnerung an den Nationalsozialismus spielen Opfer und Täter die 
Hauptrollen. ${ }^{1}$ Unter den Tätern erlangte die Figur des KZ-Arztes eine besondere Prominenz. Ihr Repräsentant ist Josef Mengele (1911-1979), der als einer von mehreren Ärzten 1943 bis 1945 im Konzentrations- und Vernichtungslager Auschwitz tätig war und im Exil der Bestrafung entging. Neben Hitler und Goebbels ist er als einer der bekanntesten Nazi-Verbrecher in die Populärkultur eingegangen: in Romane, Theaterstücke, Comics und Filme. Wie ist seine Faszinationskraft zu verstehen?

Der KZ-Arzt ist eine Symbolfigur: ein Stellvertreter des ,Bösen'. Dass Mediziner ihr berufliches Ethos, das Handeln zum Wohl der Patienten, in sein Gegenteil verkehrten, indem sie ,selektierten', experimentierten, folterten und mordeten, macht ihre Taten besonders schwer nachvollziehbar. ${ }^{2}$ Am KZ-Arzt werden die nationalsozialistischen Verbrechen im Extrem darstellbar. Fiktionale, filmische Inszenierungen der Figur entwerfen in zahlreichen Gattungen - vom historischen Drama bis zum Agenten-, Science Fiction- und Trash-Film - unterschiedliche Modelle von Täterschaft. Sie prägen so die Erinnerung und Deutung der Schoa. Dabei scheint der KZ-Arzt eine Attraktion auszuüben, die verstört und erklärt werden muss. ${ }^{3}$

Die populärkulturelle Konjunktur des KZ-Arztes im Spielfilm beginnt in den späten siebziger Jahren, kurz vor Mengeles Tod - der allerdings erst 1985 bekannt wurde (Keller 2003: 61) und auch dann noch von vielen angezweifelt wurde -, und sie reicht bis in die Gegenwart. Die Figur erfüllt dramaturgisch verschiedene Funktionen: (1.) Sie verkörpert die nationalsozialistischen Verbrechen, die überliefert und erinnert werden. (2.) Sie wird zur Projektionsfläche für Probleme der Gegenwart. (3.) Sie ermöglicht den Nachgeborenen, ihr Verhältnis zur Vergangenheit an ihr zu verhandeln. (4.) Sie wird aus dem historischen Kontext als Klischeefigur herausgelöst, die sich verselbständigt, um gattungsspezifische Funktionen in Genre-Filmen zu erfüllen.

Der folgende Aufsatz gibt einen ersten Überblick über die Filme, in denen die Figur des KZ-Arztes auftritt (als Grundlage eines weiterführenden Forschungsprojekts). Und er fragt nach ihrer jeweiligen Deutung: Wie lässt sich der KZ-Arzt als Figur des kollektiven Imaginären begreifen? Welche Topoi werden mit ihm assoziiert? Welche zeitgenössischen Probleme werden an ihm reflektiert? Welche Modelle von Täterschaft werden an der Figur entwickelt? Auf welche historischen Vorbilder beziehen sich die Filme? Und wie wird mit geschichtlichen Fakten umgegangen? Welchen Beitrag leisten die Filme zur Erinnerung des Holocaust und des Nationalsozialismus? Welche Rolle spielen

\footnotetext{
${ }^{1}$ Vgl. zur Rolle der Täter in der Gedächtnis- und Erinnerungskultur: McGothlin 2010; Adams 2015.

${ }^{2}$ Lifton spricht vom „healing-killing paradox“ (1986: 231).

${ }^{3}$ Hakes Beobachtung einer "continuing fascination with Nazi leaders, rituals, and symbols" (2012: 3), die zur Herausbildung eines "fascist imaginary in postfascist cinema“ (2012: 4) geführt habe, gilt auch und besonders für die Figur des KZ-Arztes.
} 
populäre Medien bei der Ausbildung unseres historischen Bewusstseins? Und wie ist die abseitige Anziehungskraft zu verstehen, mit der sie den KZ-Arzt in Szene setzen?

Die Präsenz, die Nazis als Filmfiguren zukommt, hat der polnische Künstler Piotr Uklánski in seiner Installation „The Nazis“ (Abb. 1) veranschaulicht, in der er 166 Aufnahmen bekannter Film-Schauspieler in NSUniformen als Portraits zusammenstellt (Uklánski 2015; vgl. Kleeblatt 2012: 108110, 82-83). ${ }^{4}$ Indem er attraktive Hollywood-Schauspieler in NS-Uniform zeigt, Nazis als Popstars, führt Uklánski vor Augen, welche abgründige Anziehungskraft von ihnen nach wie vor ausgeht.

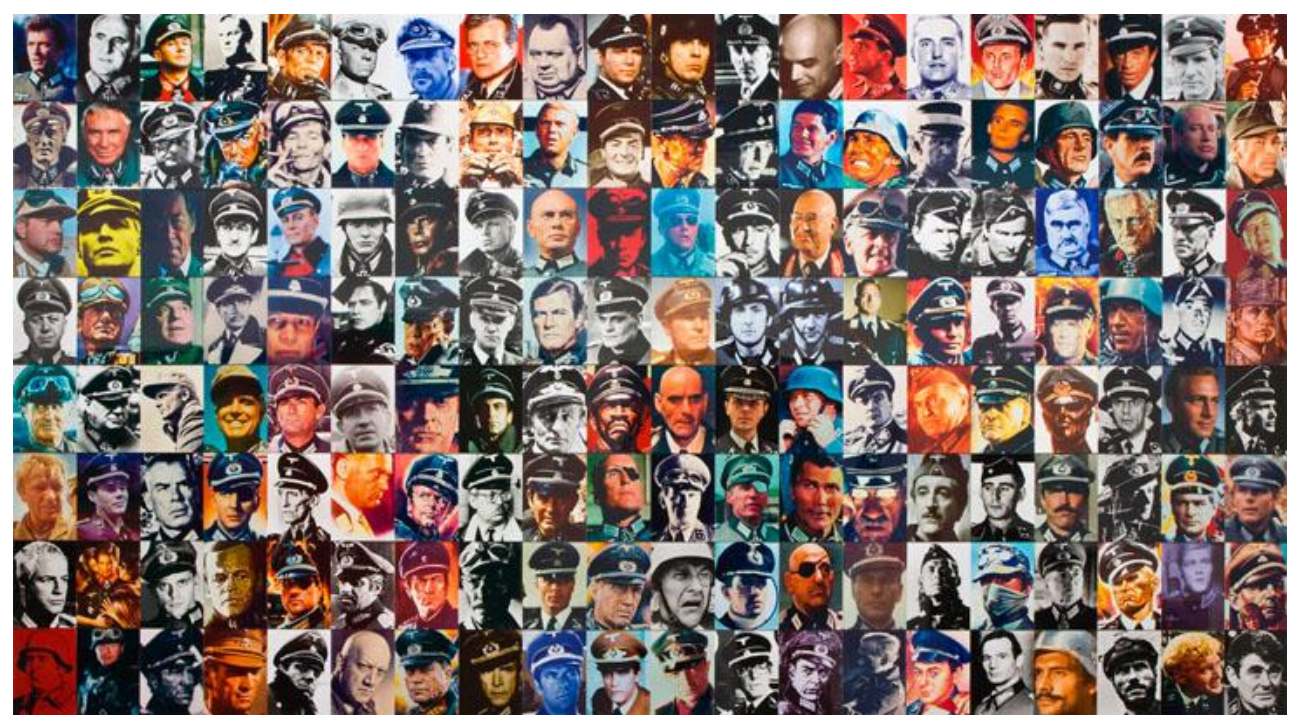

Abbildung 1: Piotr Uklánskis Installation „The Nazis“ (1998) veranschaulicht die filmische Präsenz nationalsozialistischer Figuren.

Susan Sontag (1991 [1971]) hat die Erotisierung der Nazi-Uniform prägnant als „Faszination des Faschismus“ („Fascinating Fascism“) beschrieben, die auch auf Menschen wirke, die selbst keine Nazis seien. Im Kontrast dazu steht Hannah Arendts These von der „Banalität des Bösen“ („,banality of evil“), die sie in ihrem Buch über Adolf Eichmann, den ,Buchhalter des Todes', geprägt hat (1994 [1963]). Der Massenmord war - auch - ein bürokratischer Vorgang. Und an ihm hatten Millionen gewöhnlicher Menschen teil. Wie die Filme den KZArzt innerhalb dieses Spektrums der Täterdeutungen positionieren, wird die folgende Analyse an exemplarischen Filmen untersuchen.

Die Filme, die den KZ-Arzt auftreten lassen, können thematisch und generisch in vier Gruppen eingeteilt werden: (1.) Der Täter am Tatort:

\footnotetext{
${ }^{4}$ Gezeigt wurde die Installation in der Londoner Photographer's Gallery (1998), in der Ausstellung Mirroring Evil im Jüdischen Museum New York (2002) und in der PhotographieSchau Fatal Attraction im Metropolitan Museum of Art, New York (2015).
} 
Darstellungen, die dem Genre des Holocaust-Films ${ }^{5}$ zuzuordnen sind, zeigen den Arzt im Konzentrationslager. (2.) Der Täter als Überlebender: Die Filme dieser Kategorie spielen nach 1945, sie thematisieren einerseits Flucht und Überleben des KZ-Arztes im Exil, andererseits seine Verfolgung und juristische Bewertung. (3.) Nachfahren: Filme dieser Gruppe rücken Figuren der Söhne- und Töchter-Generation und deren Verhältnis zum nationalsozialistischen Arzt in den Mittelpunkt. (4.) Action, Horror, Trash: KZ-Arzt-Darstellungen in populären Genres lösen die Figur aus ihrem historischen Kontext heraus und machen sie zu einer beliebigen und schematischen Verkörperung des Bösen.

\section{DAs MATERIAl: CHRONOlOgIE DER FILME}

Rund 30 Spielfilme, in denen die Figur des KZ-Arztes auftritt, wurden in den vergangenen fünf Jahrzehnten veröffentlicht:

- Ilsa, She Wolf of the SS, Regie: Don Edmonds, USA 1974.

- Marathon Man, Regie: John Schlesinger, USA 1976; nach dem gleichnamigen Roman von William Goldman (1974). (Mit Laurence Olivier als KZ-Arzt Dr. Christian Szell.)

- The Boys from Brazil, Regie: Franklin J. Schaffner, Großbritannien / USA 1978; nach dem gleichnamigen Roman von Ira Levin (1976). (Mit Gregory Peck als Josef Mengele.)

- Playing For Time, Regie: Joseph Sargent und Daniel Mann, USA 1980 (TV); nach der Autobiographie Sursis pour l'orchestre von Fania Fénelon (1976). (Mit Max Wright als Josef Mengele.)

- In a Glass Cage / Tras el cristal, Regie: Agustí Villaronga, Spanien 1986. (Mit Günter Meisner als Nazi-Arzt Klaus.)

- Franza, Regie: Xaver Schwarzenberger, Österreich 1986 (TV); nach dem Romanfragment Der Fall Franza von Ingeborg Bachmann (1978 [1966]). (Mit Armin Müller-Stahl als Dr. Körner.)

- The Second Victory, Regie: Gerald Thomas, Großbritannien 1986.

- Surf Nazis Must Die, Regie: Peter George, USA 1987. (Mit Michael Sonye als "Mengele".)

- Commando Mengele / The Angel of Death, Regie: Andrea Bianchi, Frankreich 1987.

- And the Violins Stopped Playing / I Skrzypce Przestaly Grac, Regie: Alexander Ramati, USA / Polen 1988. (Mit Marcin Tronski als Josef Mengele.)

- Schindler's List, Regie: Steven Spielberg, USA 1993; nach dem Roman Schindler's Ark von Thomas Keneally (1982). (Mit Daniel Del Ponte als Josef Mengele.)

\footnotetext{
${ }^{5}$ Dem Genre des Holocaust-Films lassen sich alle Filme zuordnen, welche die Verfolgung und Ermordung der Juden durch die Nationalsozialisten in historischer Perspektive darstellen (vgl. Kerner 2011: 3). Für einen Überblick zur Entwicklung des Genres vgl. Insdorf (2002: 245-249).
} 
- Nichts als die Wahrheit, Regie: Roland Suso Richter, Deutschland / USA 1999. (Mit Götz George als Josef Mengele.)

- The Grey Zone, Regie: Tim Blake Nelson, USA 2001; nach dem Zeugenbericht von Miklós Nyiszli (2001, Erstpublikation 1946 auf Ungarisch als Dr. Mengele boncoloorvosa voltam az Auschwitz-i krematoriumban). (Mit Henry Stram als Josef Mengele.)

- Amen, Regie: Constantin Costa-Gavras, Deutschland / Frankreich / Rumänien 2002; nach dem Theaterstück Der Stellvertreter. Ein christliches Trauerspiel von Rolf Hochhuth (1963). (Mit Ulrich Mühe als SS-Arzt.)

- Out of the Ashes, Regie: Joseph Sargent, USA 2003 (TV); basiert auf dem Zeugenbericht I Was a Doctor in Auschwitz von Gisella Perl (1948). (Mit Jonathan Cake als Josef Mengele.)

- My Father - Rua Alguem 5555, Regie: Egidio Eronico, Italien 2003; nach der Erzählung Vati von Peter Schneider (1987). (Mit Charlton Heston als Josef Mengele.)

- Dr. Mengele, Regie: ,Pedro', Ungarn 2005 (Porno-Film).

- החוב (deutsch: Der Preis der Vergeltung, englisch: The Debt), Regie: Assaf Bernstein, Israel 2007. (Mit Edgar Selge als Max Rainer, „Arzt von Birkenau“.)

- The Debt, Regie: John Madden, USA 2010. (Mit Jesper Christensen als Dieter Vogel, „The Surgeon of Birkenau“.)

- The Human Centipede (First Sequence), Regie: Tom Six, Niederlande 2009. (Mit Dieter Laser als Dr. Josef Heiter.)

- The Unborn, Regie: David S. Goyer, USA 2009. (Mit Braden Moran als Josef Mengele.)

- X-Men: First Class, Regie: Matthew Vaughn, USA 2011. (Mit Kevin Bacon als Dr. Klaus Schmidt.)

- Captain America: The First Avenger, Regie: Joe Johnston, USA 2011. (Mit Stanley Tucci als Dr. Abraham Erskine.)

- Nazis at the Center of the Earth, Regie: Joseph Lawson, USA 2012. (Mit Christopher Karl Johnson als Dr. Josef Mengele.)

- Wakolda - El médico alemán, Regie: Lucía Puenzo, Argentinien 2013; nach dem Roman Wakolda von Lucía Puenzo (2011). (Mit Alex Brendemühl als Josef Mengele.)

- The Ninety Nines, Regie: Howard Cook, USA 2013 (Kurzfilm, Animation).

- Im Labyrinth des Schweigens, Regie: Giulio Ricciarelli, Deutschland 2014.

- Son of Saul / Saul fia, Regie: László Nemes, Ungarn 2015. (Mit András Jeles als SS-Lagerarzt.)

\section{DER TÄTER AM TATORT}

Die filmische Inszenierung des Holocaust wird bestimmt von einem ,realistischen Imperativ‘ (Kerner 2011: 15), das heißt von dem Bemühen um eine glaubwürdige und wahrscheinliche Darstellung des Lebens und der 
Verbrechen in den Konzentrationslagern. Dies gilt auch für die hier untersuchten Filme, die (alle) verifizierbare Ereignisse darstellen und sich (in zwei Fällen) neben anderen Quellen auf Zeugenberichte von Überlebenden stützen. And the Violins Stopped Playing (USA/Polen 1988) thematisiert die Deportation polnischer Roma nach Auschwitz; The Grey Zone (USA 2001) spielt 1944 in Auschwitz und basiert unter anderem auf den Erinnerungen von Miklós Nyiszli (2001 [1946]), einem Häftling und Arzt, der zum Assistenten von Josef Mengele wurde; Out of the Ashes (USA 2003, TV) beruht auf dem Erinnerungsbuch I Was a Doctor in Auschwitz (1948) von Gisella Perl, die als rumänische Jüdin in Auschwitz interniert war, dort als ausgebildete Gynäkologin ihre Mithäftlinge betreute und Abtreibungen vornahm. Alle drei Filme beschäftigen sich mit dem zentralen Motiv des Holocaust-Films: dem Verhältnis zwischen Tätern und Opfern (Kerner 2011: 4). Bei der Darstellung des KZ-Arztes beziehen sie sich auf dasselbe historische Vorbild: den Arzt Josef Mengele, der als einer der prominentesten Nazi-Verbrecher ins kollektive Gedächtnis eingegangen ist. Mengele ist der paradigmatische Lagerarzt, obwohl er als einer von mehreren Ärzten in Auschwitz keinen herausgehobenen Rang innehatte. In den Medien der 1970er Jahre wurde er „zum ,Todesengel von Auschwitz' mythisiert und dämonisiert“" (Köhne 2013: 61). Geboren 1911, war der Erbbiologe ab 1943 - neben zahlreichen anderen Akteuren - in Auschwitz für die Selektion der Deportierten verantwortlich und führte persönlich Menschenversuche durch.

Der Heiler als Mörder steht für die Perversion der Medizin im ,Dritten Reich'. ${ }^{6}$ Als zweifach Promovierter, der Latein und Griechisch beherrschte, führt der potenzierte Akademiker als Gewaltverbrecher die Dialektik der Aufklärung vor Augen. Nach dem Krieg entkam Mengele nach Südamerika, wo er in Argentinien (1949), Paraguay (1959) und Brasilien (1960) Unterschlupf fand. Er wurde nicht, wie Adolf Eichmann (1960 in Argentinien), gefasst, sondern blieb bis zuletzt ebenso unbestraft wie uneinsichtig. Erst 1979, mehr als eine Generation nach dem Ende des ,Dritten Reiches', starb er als Achtundsechzigjähriger bei Guarujá an der Atlantikküste des Bundesstaats São Paulo beim Baden. ${ }^{7}$

\footnotetext{
${ }^{6}$ Vgl. Boozer (1980), Lifton (1986), Klee (1986, 1997, 2001), Hohendorf und Magull-Seltenreich (1990), Proctor (2000), Roelke, Hohendorf und Rotzoill (2000), Jüdisches Museum Berlin (2009), Peper und Cina (2010: 57-78), Aly (2013).

${ }^{7}$ Zur Biografie Josef Mengeles: Zdenek (1986), Völklein (1999), Posner und Ware (2000).
} 


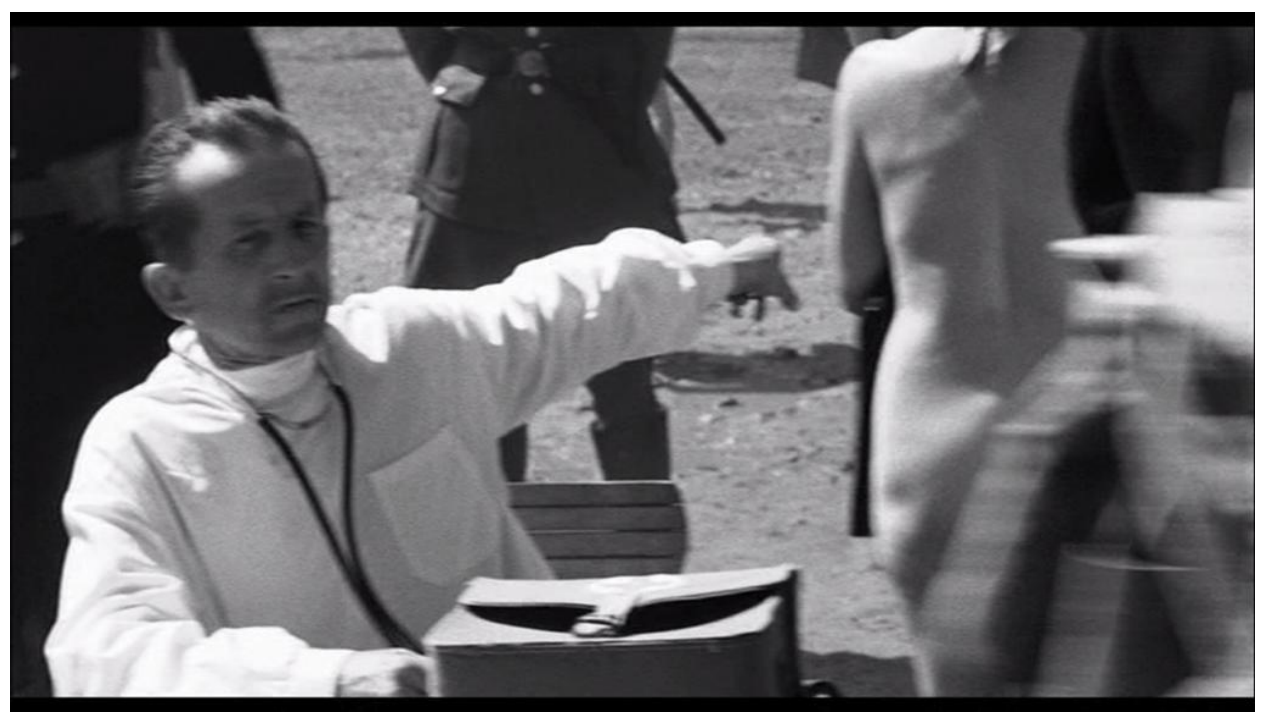

Abbildung 2: Besonders deutlich werden die Verbrechen des KZ-Arztes durch seine Beteiligung an der Selektion - eine Situation, die zahlreiche Filme inszenieren, hier ein Standbild aus Schindler's List (USA 1993).

Mit Josef Mengele als Stellvertreter der Täter wählen die Filme einen Protagonisten, der durch seine Beteiligung an der Selektion in Auschwitz, dem bekanntesten Konzentrationslager, an der systematischen, rassistisch motivierten Ermordung von Millionen Menschen durch die Nationalsozialisten beteiligt war, und zwar in deren vorletzter Phase, der Entscheidung über Leben und Tod. Er wird so zur Verkörperung der Vernichtungsmaschinerie. Seine Selektionstätigkeit bei der Ankunft der Züge in Auschwitz, die er distanziert und emotionslos ausführt, wird in den Filmen häufig in Szene gesetzt (Abb. 2). Besonders beklemmend inszeniert ist die Situation in Out of the Ashes (Abb. 3): Die Szene zeigt Mengele scheinbar unbeteiligt, klassische Melodien pfeifend, während er mit einem Fingerzeig seiner weiß behandschuhten Hand, die unscharf in den Bildvordergrund ragt und über dem Kopf der Betrachtenden zu schweben scheint, die Deportierten für Zwangsarbeit und Menschenversuche auswählt oder in die Gaskammern schickt.

Die Grausamkeit seiner Menschenversuche stellt besonders eindringlich die Frage nach Mengeles Antrieb: Handelte er aus ideologischer Überzeugung, aus wissenschaftlichem Ehrgeiz oder aus sadistischer Perversion? Die Holocaust-Filme schreiben ihm in der Regel eine Kombination dieser unterschiedlichen Motivationen zu. Häufig wird ihm ein manischer ForscherEhrgeiz attestiert, während seine ideologisch verklärten Ziele als wissenschaftlich abwegig charakterisiert werden. ${ }^{8}$ In Out of the Ashes ist Mengele laut eigener Aussage auf der Suche nach dem ,magischen Geheimnis'

\footnotetext{
${ }^{8}$ Die Figur steht so in der Tradition von H. G. Wells' besessenem Dr. Moreau (2005 [1896]), der in seinem Labor Tiere zu Menschen umzuoperieren versucht, ohne dass sich damit ein medizinisches Ziel verbindet. Vgl. zur Motivtradition des folternden und mordenden Arztes Gomel 2000.
} 
von Zwillingsgeburten, um so die Zahl der Angehörigen der ,arischen Rasse' multiplizieren zu können. Als Wissenschaftler disqualifiziert er sich hier nicht nur durch seine brutalen Methoden und sein rassistisches Ziel, sondern auch durch sein uninformiertes Forschungsprogramm: Im Film wendet die jüdische Ärztin ein, bei der Zwillingsempfängnis handele es sich keineswegs um ein ,magisches‘ Ereignis, sondern um einen längst erforschten biologischen Prozess. Das Forschungsprojekt zeigt so Mengeles ideologische Verblendung und mangelnde Fachkompetenz. In And the Violins Stopped Playing versucht er, braune in blaue Augen zu verwandeln, was durch die skeptische Nachfrage eines Mitarbeiters (,What is the scientific benefit of your discovery?") als unwissenschaftliches und unsinniges Ziel gekennzeichnet wird. ${ }^{9}$

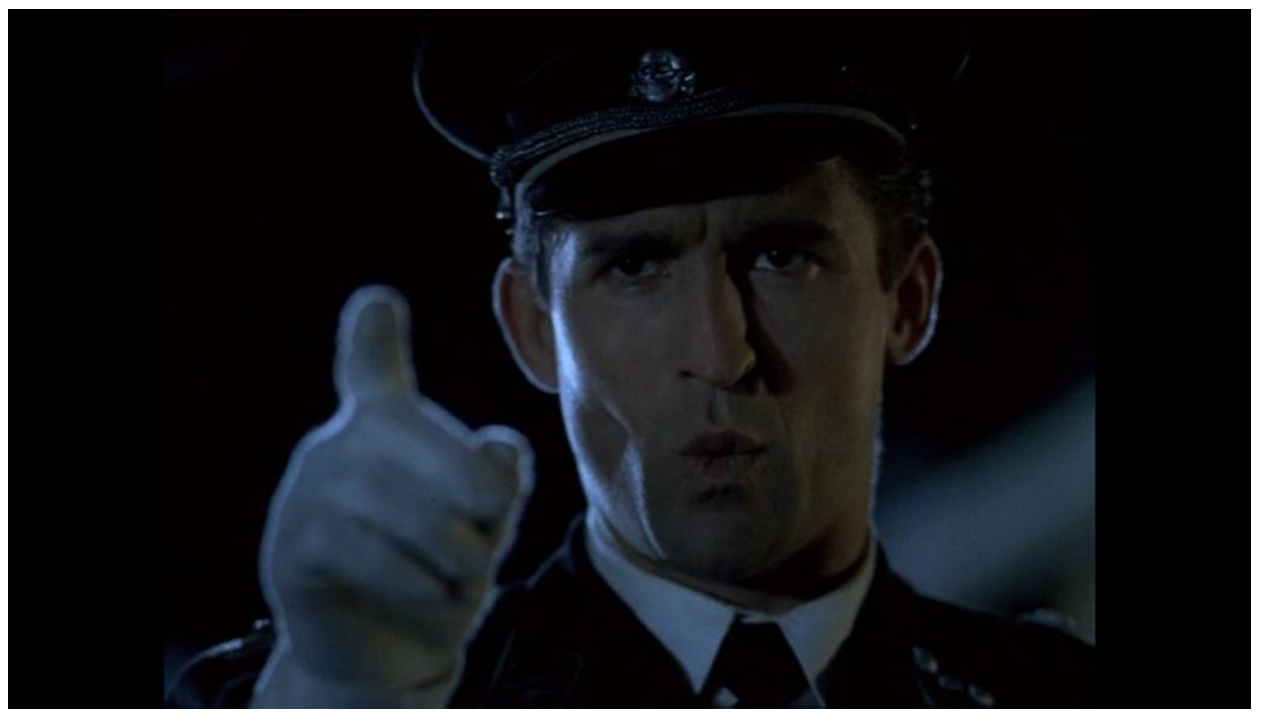

Abbildung 3: Mengele bei der Selektion in Out of the Ashes (USA 2003).

Die Grausamkeit seines Vorgehens wird von den Filmen meist mit Mengeles sadistischem Charakter erklärt. Er handele zu seinem perversen Vergnügen, vermutet Gisella Perl in Out of the Ashes. In diesem Film zeichnet er einer schwangeren Frau eine Skizze auf den Bauch, die ihm nach der Geburt ihrer Kinder und ihrer darauf folgenden Ermordung bei der Autopsie behilflich sein soll.

Mit der Figur Josef Mengeles greifen die Filme neben dem rassistischen Massenmord und der Frage nach der Tätermotivation ein weiteres zentrales Thema der Holocaust-Erinnerung auf: den vermeintlichen Widerspruch zwischen den Verbrechen und der Bildung, Kultur und ,Zivilisation' vieler Nazitäter (Kerner 2011: 47f.). So wird Mengele sowohl in Out of the Ashes als auch in The Violins Stopped Playing als durchaus einnehmende Person dargestellt, die dann emotionslos Gewalttaten begeht. Charakteristische Topoi,

9 Dass Mengeles Vorhaben die von den Nazis postulierten ,Rassen-Unterschiede' in Frage stellt bzw. anders als in der nationalsozialistischen Theorie auf einer lediglich phänotypischen Ebene verortet, macht es zudem widersprüchlich und führt es in sich selbst ad absurdum. 
die seine oft tödlichen Menschenversuche um so grausamer erscheinen lassen, sind seine Freundlichkeit insbesondere gegenüber Kindern, seine Höflichkeit, ein charmantes Auftreten und kulturelle Bildung. ${ }^{10}$ Mengele provoziert die sprichwörtliche Frage, die Alfred Andersch in seiner "Schulgeschichte“ Der Vater eines Mörders (1982 [1980]), in der Erinnerung an eine Griechisch-Stunde bei dem autoritär-sadistischen Vater von Heinrich Himmler, gestellt hat (und auch sich selbst stellen musste): „Schützt Humanismus denn vor gar nichts?“ (1982: 136) Glaubt man den Filmen und ihrer Inszenierung Mengeles, lautet die Antwort: Nein, ganz offenbar nicht.

Zwei Holocaust-Filme zeichnen sich durch eine besondere Figurenkonstellation aus. The Grey Zone und Out of the Ashes lassen neben Mengele eine weitere Arzt-Figur auftreten, die unter den Häftlingen rekrutiert wurde: einen jüdischen KZ-Arzt bzw. eine jüdische KZ- ̈̈rztin. Diese Konstellation verkompliziert das Verhältnis zwischen Opfern und Tätern, das in The Violins Stopped Playing noch traditionell manichäisch angelegt war. Die in der Nachkriegszeit spielende Rahmenhandlung von Out of the Ashes macht dies besonders deutlich. Die nach ihrer Befreiung aus dem Konzentrationslager in die USA emigrierte Gynäkologin Gisella Perl hat die Prüfung des New York State Medical Licensing Board bereits bestanden, benötigt jedoch die USamerikanische Staatsbürgerschaft, um wieder als Ärztin praktizieren zu dürfen. Als sie deshalb beim Immigration $\mathcal{E}$ Naturalization Service vorspricht, wird sie mit dem Vorwurf konfrontiert, im KZ mit den Nationalsozialisten kollaboriert zu haben. Auch dass sie dort Abtreibungen vorgenommen hat, wird ihr zur Last gelegt (erst 1970 wurde in den USA die Abtreibung auch jenseits der Einzelfallerlaubnis legalisiert). Die Binnenerzählung über Gisella Perls Erlebnisse in Auschwitz dient dann ihrer Rehabilitierung: Wie der Film deutlich macht, hat sie sich von Mengeles freundlichem Auftreten zunächst täuschen lassen und sich nach ihrer Einsicht in seine Brutalität nur unter Zwang und um ihre Mitgefangenen zu schützen als Ärztin zu erkennen gegeben. Gerade die Abtreibungen, die ihr vorgeworfen werden, führen Mengeles provokative Behauptung, er und Perl seien einander ähnlicher, als sie sich eingestehen wolle, ad absurdum. Während Mengele Perls Deutung zufolge einerseits aus Vergnügen, andererseits im Dienst fragwürdiger wissenschaftlicher Zwecke tötet, nimmt sie die Abtreibungen vor, um Leben zu retten, denn Schwangere hatten in Auschwitz wenig Chancen zu überleben. Der Film macht deutlich, dass Perls Verstöße gegen den Hippokratischen Eid (den sie zitiert) innerhalb des Konzentrationslagers keineswegs als Verbrechen zu verstehen sind, die sie mit Mengele vergleichbar machen würden. Indem sie in der Schlussszene einer Frau, deren erstes Kind sie in Auschwitz abgetrieben hatte, bei der Geburt

\footnotetext{
10 Besonders deutlich wird dieser Kontrast von ,Zivilisation' und ,Barbarei' auch in Playing for Time (USA 1980, TV) inszeniert, einem Film über das Mädchenorchester in Auschwitz, der Mengele als Liebhaber klassischer Musik zeigt.
} 
assistiert, betont der Film ihren Gegensatz zu Mengele, der hier wiederholt als ,Dr. Death' apostrophiert wird. Am Ende des Films wird so die Dichotomie von Tätern und Opfern, die in seinem Verlauf fraglich zu werden schien, wieder hergestellt.

Anders verhält es sich in The Grey Zone. Das titelgebende Thema ist neben dem nationalsozialistischen Massenmord in Auschwitz die moralische 'Grauzone', in der diejenigen Häftlinge handeln, die, um sich selbst zu retten, mit den Nationalsozialisten kooperieren (Bangert 2008: 20). ${ }^{11}$ Der Film bezieht sich auf einen gleichnamigen Text von Primo Levi (1993 [1986]), der die Problematik der erzwungenen Kollaboration beschreibt und eine „impotentia judicandi“ (Levi 1993 [1986]: 60) konstatiert: die Unmöglichkeit, das Verhalten der Betroffenen zu beurteilen. ${ }^{12}$ Die Handlung des Films spielt im Jahr 1944. Sie entfaltet das Thema einerseits an einem in den Gaskammern und Krematorien tätigen Sonderkommando und andererseits durch die Darstellung des jüdischen Arztes Miklós Nyiszli. Durch seine Tätigkeit für Mengele hofft Nyiszli, seine Frau und Tochter retten zu können, aber er wird so unfreiwillig zum Mittäter. ${ }^{13}$ Die Figur des jüdischen Arztes eignet sich für die Darstellung des ethischen Dilemmas der KZ-Häftlinge, ${ }^{14}$ weil sie, wie McGothlin ausführt, den Topos des Arztes als "heroic agent of rescue“ (2015: 191) zugleich aufruft und unterläuft und damit die Deheroisierung der Figuren auf die Spitze treibt.

Den Film eröffnet eine Szene, in der Nyiszli einen Häftling nach einem Mordversuch durch einen Mitgefangenen wiederbelebt. Diese auf den ersten Blick ehrenwerte Erfüllung der wichtigsten ärztlichen Aufgabe - der Lebensrettung - stellt sich im Lauf des Films aber als fragwürdig heraus, da sie womöglich nicht im Sinn des ,Patienten' war (McGothlin 2015: 192): Denn der Betroffene ist Mitglied des Sonderkommandos und hatte, wie die ZuschauerInnen später erfahren, die Leichen seiner Angehörigen im Krematorium verbrennen müssen. In seiner Verzweiflung hatte er sich den von Nyiszli verhinderten Tod gewünscht. Deutlich wird so, dass gängige ethische Bewertungen im Kontext des Konzentrationslagers nicht anwendbar sind: „In Auschwitz, in which the medical ethics in particular are overturned, Nyiszli's rescue of the prisoner [...] and his professional code of medical ethics [...] are viewed by the Sonderkommando prisoners as a ludicrous remnant of an irrelevant moral realm." (McGothlin 2015: 193) Die nachträgliche ReEvaluierung der Anfangsszene, welche die ZuschauerInnen vornehmen müssen - der vermeintliche Mordversuch erscheint retrospektiv als "charitable act" (Pugliese 2003: 958), die Rettung durch den Arzt als problematische

\footnotetext{
${ }^{11}$ Neben seiner metaphorischen Bedeutung (der moralischen Grauzone) bezieht sich der Titel auf die graue Asche der ermordeten und verbrannten Gefangenen (vgl. Boswell 2012: 159f.).

${ }^{12} \mathrm{Zu}$ Levis Text Die Grauzone vgl. u.a. Henry (2009).

13 "Without you his research would be impossible", wirft ihm ein Mithäftling vor.

${ }^{14}$ Treffend paradoxiert Baron die Entscheidungen der Häftlinge als „choiceless choices“ (2005: 286).
} 
Intervention -, macht deutlich, wie wenig sich das Verhalten der Häftlinge beurteilen lässt. Zwar wird der KZ-Arzt Mengele klar als verbrecherischer Täter identifiziert. In den Fokus rückt der Film jedoch die verzweifelte Situation der Figuren, die in der ,Grauzone' agieren. Auschwitz wird als ein Raum dargestellt, der keinerlei „virtuous action“ (Boswell 2012: 168) zulässt und in dem eines der Verbrechen gerade darin besteht, dass die Nationalsozialisten ihre Gefangenen zwingen, selbst zu Mittätern zu werden.

Der Auftritt des Täters am Tatort erfüllt in den Holocaust-Filmen somit dramaturgisch drei Funktionen: Er erinnert an die grausamen Verbrechen der Nationalsozialisten in den Konzentrationslagern; er hinterfragt die Motivation für diese Taten, die in scheinbarem Widerspruch zu Bildung und Kultiviertheit der Täter stehen; und schließlich problematisiert er das ethische Dilemma der erzwungenen Mittäterschaft der Inhaftierten.

\section{DER TÄTER ALS ÜBERLEBENDER}

Die unerträgliche Provokation, die Mengele im Unterschied $\mathrm{zu}$ anderen bekannten Nazi-Tätern auslöst, besteht auch darin, dass er seine Verbrechen ohne Strafe und ohne Reue überleben konnte. Eine zweite Reihe von Filmen thematisiert das Über- und Weiterleben der Täter nach 1945 anhand von KZArzt-Figuren, die der Bestrafung entgehen und in der Gesellschaft der Nachkriegszeit untertauchen. Ihre Figuren stehen für das heimliche bzw. unheimliche Fortleben nationalsozialistischer Ideologie. Sie verkörpern die andauernde Gefahr des Faschismus und zugleich die Furcht vor der eigenen Anfälligkeit. Das ,Böse', das der unentdeckte Nazi verkörpert, ist buchstäblich unter uns, vielleicht sogar in uns.

Anders als die Holocaust-Filme, deren Handlungen in der historischen Zeit des Nationalsozialismus spielen, steht den Filmen, in denen der KZ-Arzt nach 1945 auftritt, ein größerer Spielraum für den Entwurf fiktiver oder fiktionalisierter Figuren und Geschehnisse zur Verfügung. Sie thematisieren häufig nicht ein überliefertes historisches Ereignis, sondern entwickeln kontrafaktische Plots, die sie in Genres wie der Science Fiction, dem Thriller oder dem Justizdrama in Szene setzen. Die Handlung dieser Filme spielt in der Regel in der Zeit ihrer Entstehung, also in der Gegenwart der ZuschauerInnen, die den Film im Kino sehen. Auf diese Weise findet nicht nur eine Aktualisierung der historischen Figur statt - sie agiert in der Gegenwart und rückt so in größere Nähe -, sondern sie dient darüber hinaus regelmäßig auch der Verhandlung zeitgenössischer Probleme und Fragestellungen. Der KZ-Arzt wird zur Projektionsfigur für Konflikte und Themen der Nachkriegszeit. ${ }^{15}$

${ }^{15}$ Auch hier lässt sich an der Figur des KZ-Arztes aufzeigen, was generell für die fiktionale Darstellung von Nationalsozialisten gilt: „filmic representations of Nazism/fascism have provided a projection screen for the problems of postwar democracies" (Hake 2012: 4). 
The Boys from Brazil (USA 1978) inszeniert den geflohenen Josef Mengele als akute Bedrohung. (Mengele hätte diesen Spielfilm theoretisch selbst noch im Kino sehen können.) Mengele arbeitet, so der Plot, im südamerikanischen Exil buchstäblich an einer Wiederbelebung nationalsozialistischer Herrschaft: Er hat, wie der Film schrittweise enthüllt, Adolf Hitler zu Lebzeiten genetisches Material entnommen, aus dem er in den 1960er Jahren in Paraguay 94 Klone schuf. Diese wurden von Leihmüttern ausgetragen und anschließend weltweit an ausgewählte Familien zur Adoption vermittelt. Als die Filmhandlung einsetzt, sind die Hitler-Klone 13 Jahre alt und haben damit das Alter erreicht, in dem der historische Hitler seinen Vater verlor. Da der Film-Mengele annimmt, dass nicht nur die Gene, sondern auch die Sozialisation und die Lebensgeschichte eine Persönlichkeit prägen, beauftragt er alte und neue Nazis aus dem Kreis einer geheimen ,Comrade Organization', die 94 Adoptivväter der Hitler-Klone umzubringen. Auf diese Weise, so hofft er, erhöhen sich die Chancen, dass sich unter den Heranwachsenden eine Persönlichkeit herausbildet, die als ,Führer' eine neue nationalsozialistische Herrschaft, ein ,Viertes Reich' begründen kann: „the right Hitler for the right future; a Hitler tailor-made for the 1980s, 90s, 2000s."

Als Gegenspieler lässt der Film Ezra Liebermann (gespielt von Laurence Olivier) auftreten, der dem ,Nazijäger' Simon Wiesenthal nachempfunden ist. Während Mengele auf ein funktionierendes Netz nationalsozialistischer Gesinnungsgenossen zurückgreifen kann und mit finanziellen Mitteln ausgestattet ist, wird Liebermann als zerstreuter, bisweilen komisch wirkender Einzelkämpfer gezeichnet, der weder über materielle noch über institutionelle Unterstützung verfügt.

Schaffners Film entwirft ein bedrohliches Szenario von der Wiederkehr des Faschismus, das zeitgenössische kollektive Ängste aufnimmt. Der flüchtige Mengele übt nicht nur als überlebender und unauffindbarer Nazi-Verbrecher und damit als potentieller neuer Anführer nationalsozialistischer Herrschaftsanpläne gleichermaßen Schrecken und Faszination aus, er praktiziert mit dem genetischen Klonen zugleich eine in den 1970er Jahren kontrovers diskutierte und angstbesetzte neue medizinische Technik (Köhne 2013: 63). Indem ihm das Klonen eines Menschen (sogar gleich seriell) gelingt, ist er der institutionellen Forschung einen Schritt voraus. Der Film, der Merkmale der Genres des Horror-, Kriminal- und Science-Fiction-Kinos kombiniert, inszeniert Mengele als mad scientist, ${ }^{16}$ der aktuelle biotechnologische Verfahren genial weiterentwickelt, um seine Ideologie zu verwirklichen.

${ }^{16}$ Vgl. Köhne (2013: 66); zur Figur des mad scientist vgl. Stiles (2009). 


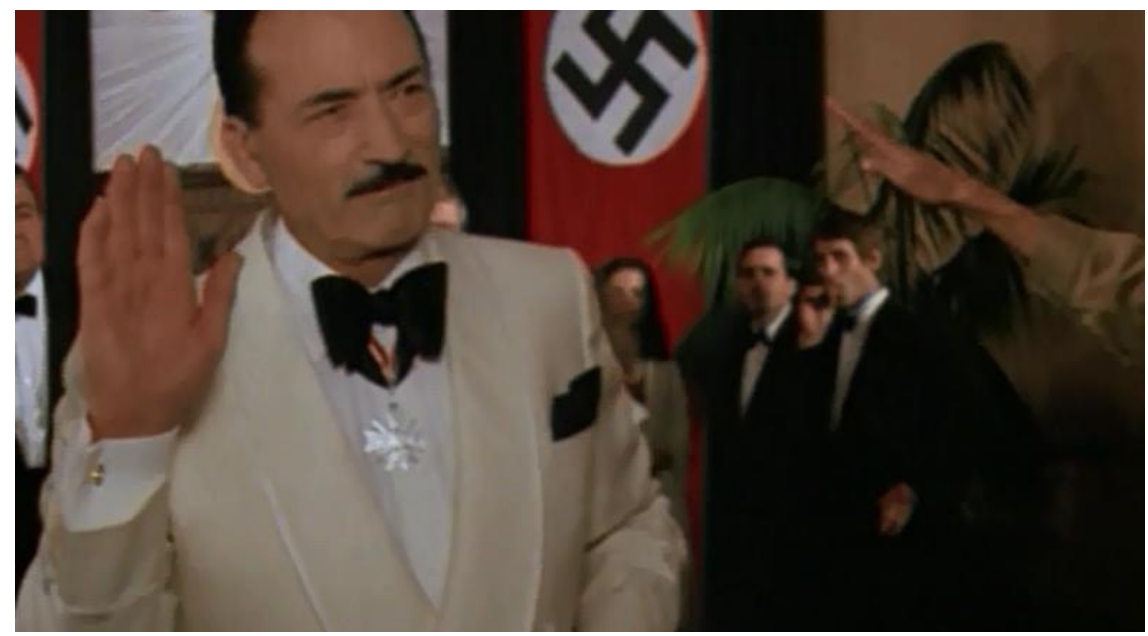

Abbildung 4: Der lässige ,Führergruß’ unterstreicht Mengeles Rolle als Führungspersönlichkeit in der nationalsozialistischen Geheim-Organisation in The Boys from Brazil (USA 1978).

Optisch inszeniert der Film Mengele als elegante, ehrfurchtgebietende und charismatische Figur. Seinen verzögerten Auftritt in der 13. Minute kündigen mehrere Vorausdeutungen an, welche die Erwartung steigern. Angestrahlt von Scheinwerferlicht, entsteigt er in einem auffällig weißen Anzug, gleichsam epiphanisch, einem Flugzeug (Abb. 5). In einer vergleichbaren Szene erscheint er auf einem Pferd.

Die Sequenz präsentiert ihn aus der Untersicht - eine Kameraperspektive, die in der filmischen Darstellung Mengeles wie auch der Hitler-Klone (vgl. Abb. 6) wiederholt zum Einsatz kommt und die Bedrohlichkeit, aber auch die Faszination der Figur visuell in Szene setzt: Mengele wird buchstäblich überhöht. Auch die Starbesetzung mit Gregory Peck trägt zu dieser Wirkung bei.

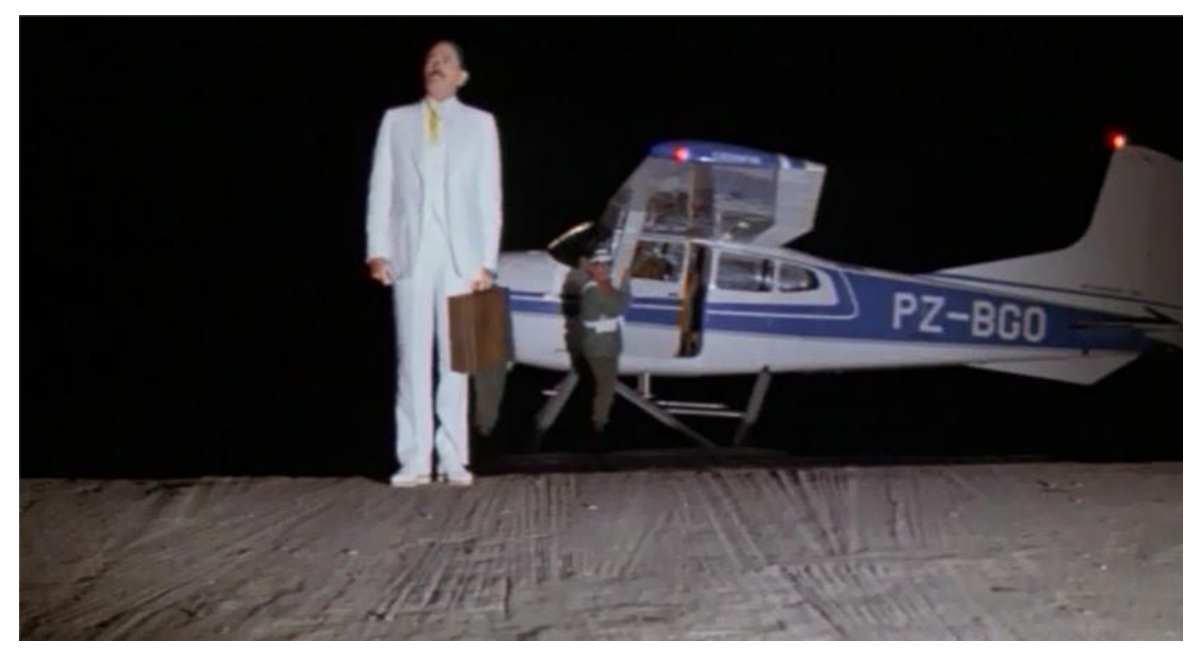

Abbildung 5: Wie eine Epiphanie mutet Mengeles erster Auftritt in The Boys from Brazil (USA 1978) an, als er im weißen Anzug und von Scheinwerfern angestrahlt einem Flugzeug entsteigt. 
Im Kontrast zu ihrer Eleganz steht die kaltblütige Brutalität der Figur. Für den Erfolg seiner „holy mission“ schreckt er nicht vor dem Mord an Kindern oder auch an Gesinnungsgenossen zurück. Dabei wird er - der Unterstützung seiner Mitstreiter beraubt - schließlich auch eigenhändig zum Mörder. Der Film endet mit einem Showdown in Pennsylvania: Im Haus der Familie eines seiner HitlerKlone treffen Mengele und Liebermann aufeinander - Mengele in der Absicht, sein Projekt mordend zu Ende zu führen, Liebermann in der Hoffnung, ihn daran zu hindern.

Der Hitler-Klon Bobby Wheelock (wie alle Klone gespielt von Jeremy Black) trifft auf die Kontrahenten im Wohnzimmer seiner Familie. Als er erfährt, dass Mengele seinen Adoptivvater ermordet hat, hetzt er die Dobermänner der Familie auf ihn und sieht fasziniert dabei zu, wie diese ihn zerfleischen.

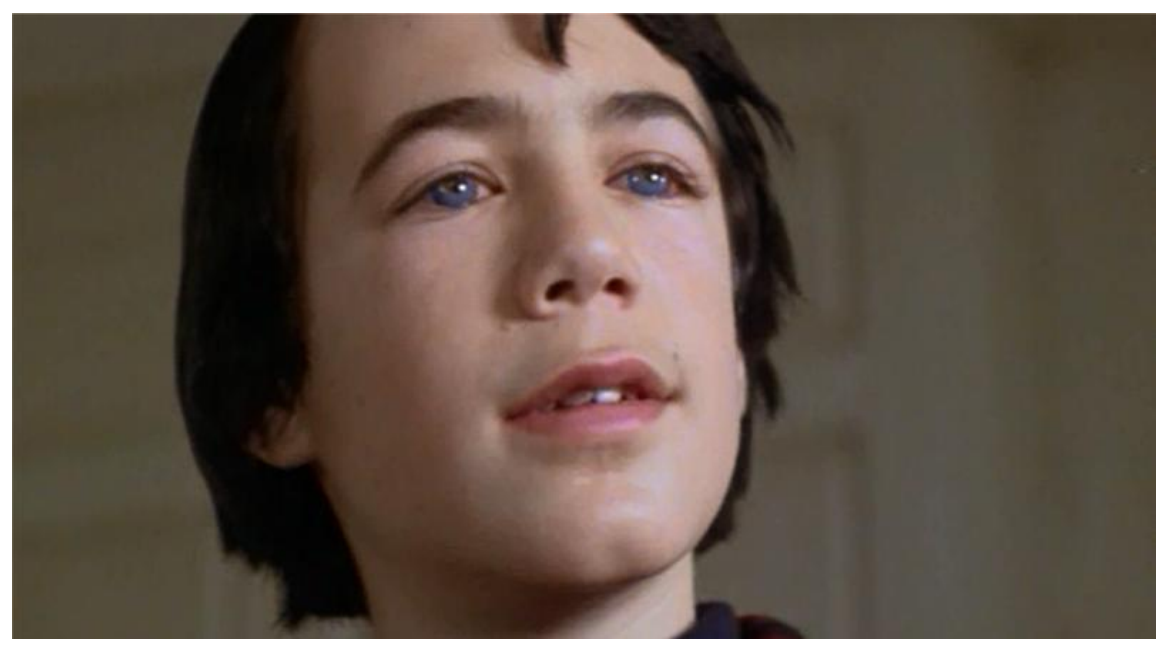

Abbildung 6: Der Hitler-Klon Bobby Wheelock in The Boys from Brazil (USA 1978) wird seinerseits präsentiert aus der Untersicht und mit auffällig blauen Augen - ein häufiges Motiv in den Filmen, die KZ-Arzt-Figuren auftreten lassen.

In Anlehnung an bekannte Narrationen der künstlichen Erschaffung von Lebewesen - insbesondere Mary Shelleys Frankenstein (2012 [1818]) - wird der Schöpfer zum Opfer seines Geschöpfs. ${ }^{17}$ Zugleich bestätigt jedoch gerade diese Szene paradoxal Mengeles Erfolg: Bobby Wheelock hat mehrere Eigenschaften, die ihn als Hitler-Figur ausweisen und die vermuten lassen, dass er die Aufgabe, für die ihn Mengele vorsah, erfüllen kann; auffällig sind besonders

17 Das Konzept des Klons, eines künstlich erschaffenen Menschen, ist mit verschiedenen "Traditionslinien“ dieses Motivs verknüpft, wie Marek ausführt, unter anderem einer "magisch-mythische[n] (Golems, Homunculi, belebte Statuen etc.)" sowie einer "mechanischelektrische[n] (die Automatenwesen des späten 18. Jahrhunderts, etwa die Olimpia-Puppe, sowie chirurgisch zusammengeflickte Kunstmenschen des späten 19. Jahrhunderts à la Frankenstein“ (2012: 19). Als gefährliche Konsequenz der selbstermächtigten künstlichen Erschaffung lebender Wesen drohen in vielen Erzählungen dieses Motivs „Kontrollverlust" und „Verselbständigung“ (Marek 2012: 22). 
seine Gewaltbereitschaft, sein Selbstbewusstsein und seine künstlerischen Ambitionen (als Fotograf). Die Schlussszene legt nahe, dass Mengeles Projekt eines ,Vierten Reiches' posthum realisiert werden kann. Sie zeigt Bobby Wheelock mit Mengeles Haifischzahnkette, die er offenbar an sich genommen hat. Mit einer Großaufnahme dieser Kette endet der Film. Er signalisiert mit diesem prominent eingesetzten symbolischen Artefakt, dass Bobby Wheelock das ,Erbe' Mengeles - und zugleich Hitlers - antritt.

The Boys from Brazil genialisiert, charismatisiert und überhöht also den KZArzt. Mengele tritt als eleganter Nazi auf, der als hochbegabter mad scientist ein angstbesetztes wissenschaftliches Verfahren erfolgreich entwickelt und in den Dienst nationalsozialistischer Ambitionen der (Welt-)Herrschaft stellt. Und er erscheint sogar gottähnlich: Hitlers Genmaterial hat er ausgerechnet aus dessen Rippe entnommen und so den göttlichen Schöpfungsakt imitiert. Obwohl oder gerade weil der ,Schöpfer' Mengele schließlich von seinem Geschöpf getötet wird, zeichnet der Film ihn als eine mächtige, dämonische Figur, der es mit seiner Wissenschaft gelingt, ,das Böse' in die Welt (zurück) zu bringen. Seine Motivation wird dabei eindeutig als ideologische gekennzeichnet: nicht Geldgier oder Erkenntnisdrang sind die Triebkraft seiner Handlungen, sondern der Glaube an die Vorzüge der ,arischen Rasse' und die Hoffnung auf die nationalsozialistische Weltherrschaft. Damit charakterisiert der Film seine Mengele-Figur als „Weltanschauungstäter" (Paul 2002: 61).

Indem er die Figur des Nazi-Arztes mit der Thematik des Klonens verbindet, liefert der Film zugleich einen diskursiven Beitrag zur GentechnikDebatte (Wulff 2002: 211). Zwar behauptet er nicht, das Klonen sei per se ,böse', er assoziiert diese Technik jedoch "mit der dystopischen Vision einer Reaktivierung nationalsozialistischer Machtinteressen sowie von Rassenhygiene und Eugenik" (Köhne 2013: 56). ${ }^{18}$ Die Mengele-Figur wird so aktualisiert: Sie wird zum Vehikel einer fiktionalen Auseinandersetzung mit einer wissenschaftskritischen Debatte, die, als der Film entstand, intensiv geführt wurde. Und auch politisch wird das Thema aktualisiert: Die ,Wiederbelebung“ Hitlers („Hitler is alive“, heißt es im Film) wird zu einer weltweiten Bedrohung, die durch die Konzentration auf Bobby Wheelock - kurz nach dem Ende des Vietnamkrieges - dieses Mal nicht von Österreich oder Deutschland, sondern von den USA ausgeht.

In Marathon Man (USA 1976) wird der KZ-Arzt ebenfalls als Drahtzieher in Szene gesetzt, er tritt hier jedoch nicht als ideologisch überzeugter Nazi auf. Statt durch rassistische Motive sind seine Handlungen hier durch Geldgier begründet: In Auschwitz hatte Szell seinen Opfern Diamanten abgepresst, die nun bei einer Bank in New York lagern und ihm bis zum Einsetzen der

18 Vgl. auch: „Die zeitgenössisch empfundene Bedrohung hyperpotenter wissenschaftlicher Neuerungen wird im Filmplot mit narrativen Elementen des nationalsozialistischen Genozids gekoppelt.“ (Köhne 2013: 56) 
Filmhandlung mit der Hilfe seines Bruders und, wie sich nach und nach erschließt, des US-amerikanischen Geheimdienstes in sein Exil in Uruguay geschleust wurden. Der Tod seines Bruders in der Eröffnungsszene zwingt Szell, selbst nach New York zu reisen um die Diamanten aus dem Schließfach $\mathrm{zu}$ holen. Anders als in den bisher thematisierten Filmen handelt es sich bei dem KZ-Arzt in Marathon Man also - nach der Tätertypologie von Paul (2002: 61) - nicht um einen Weltanschauungstäter, sondern um einen utilitaristisch motivierten Täter.

Auch Szells Auftritt wird effektvoll hinausgezögert, sogar bis zur 37. bzw. 54. Minute (zuerst erscheint er indirekt, als Photo, dann selbst, als Akteur). Abermals ist die Figur elegant gekleidet: im grauen Dreiteiler, mit Krawatte alle Insignien, die ihn als Nationalsozialisten identifizieren würden, fehlen jedoch. Und erneut ist die Figur mit einem Star-Schauspieler besetzt - Laurence Olivier, der in The Boys from Brazil Mengeles Antagonisten spielt. Der Vorname der Figur, Christian Szell, verweist auf Christus. "Der weiße Engel“ von Auschwitz steht für eine Metaphysik des Bösen - als gefallener ,Engel', Lucifer, der ,das Licht bringt' und hier sein Opfer bei der Folter (als Zahnarzt) mit einer Verhörlampe blendet.

Szells jüdischer Gegenspieler, „Babe“ Levy (Dustin Hoffman), trägt seinerseits die Unschuld im Vor- bzw. Spitznamen und sein Judentum im Nachnamen. Zu Szells Verfolger wird er nur zufällig und unfreiwillig: Sein Bruder, der die Deck- bzw. Spitznamen „Scylla“ und „Doc" trägt, arbeitet für Szell - bzw. für die „Division“, eine Institution der Regierung, die Szell beim Diamantenschmuggel unterstützt, weil dieser als Gegenleistung die Aufenthaltsorte von Nazis verrät. Bei einem Streit wird Scylla von Szell ermordet, er kann jedoch vor seinem Tod noch Babes Wohnung erreichen. In der Annahme, Scylla habe Babe geheime Informationen anvertraut, lässt Szell diesen durch seine Helfer Karl und Erhard entführen. Er foltert ihn, und zwar nach der Logik der Perversion der Medizin im ,Dritten Reich' durch eine ,Zahnbehandlung', die er zwar durch das Auslegen seiner Instrumente und das Desinfizieren seiner Hände medizinisch vorbereitet, die dann jedoch lediglich dazu dient, seinem Opfer Schmerzen zuzufügen.

Gerettet wird Babe - scheinbar - von Peter Janeway, einem weiteren USGeheimagenten, der ihn jedoch, als er dessen Fragen nicht beantworten kann, wiederum Szell und der Folter ausliefert. Für Babe und die ZuschauerInnen erschließt sich erst in diesem Moment die Zusammenarbeit zwischen dem (Ex)Nazi und dem US-amerikanischen Geheimdienst, der dessen Methoden offenbar fraglos akzeptiert. War der KZ-Arzt in den bisher thematisierten Filmen jeweils die bedrohlichste Figur, so entfaltet sich die Bedrohung als beklemmendes Gefühl der Ausweglosigkeit hier wesentlich aus der Kooperation zwischen Szell und den US-Amerikanern. Dass Babe nicht nur von Szell verfolgt wird, sondern darüber hinaus auch den Staatsangestellten nicht vertrauen kann, die eigentlich für seinen Schutz zuständig wären, bildet die 
überraschende Wendung des Films, der damit die Rolle des US-amerikanischen Geheimdienstes mindestens im gleichen Maß thematisiert und problematisiert wie die Verbrechen des deutschen Nazi-Arztes. In einer unerwarteten Umkehrung bekannter Verhältnisse ist der KZ-Arzt nicht Feind der USamerikanischen Agenten, sondern Komplize. (Vergleichbar etwa dem Raketeningenieur Wernher von Braun, der von der NASA übernommen wurde.) Für Babe werden beide Parteien gleichermaßen zu gefährlichen Gegnern: In einem Feuergefecht mit Janeway tötet er diesen in Notwehr, nachdem ihm Elsa, seine Geliebte und zugleich Mitarbeiterin Szells - in einer weiteren ironischen Wendung, welche die Zugehörigkeiten verunklart - das Leben gerettet hatte.

Weiter hervorgehoben wird die überraschende Kollaboration zwischen dem NS-Kriegsverbrecher und den US-Amerikanern durch eine Reihe von Gemeinsamkeiten zwischen Szell und den Geheimagenten. So ist Scyllas mythologisch inspirierter Deckname nicht nur klangähnlich mit Szell, sondern er deutet auch auf die Gemeinsamkeit (und zugleich subtil auf die Gegensätzlichkeit) der beiden Figuren: als Scylla und Charybdis. Weiter unterstrichen wird die Gemeinsamkeit dadurch, dass beide - zumindest dem (Spitz-)Namen nach - „Doc“ bzw. Doktor sind. Auch die Kleidung des Protagonisten wie des Antagonisten dient ihrer Annäherung: Szell und seine US-amerikanischen Partner teilen "the cold professional look of a typical businessman" (Gross 1985: 58). ${ }^{19}$

Dass Babe zwar Jude ist, seinen Vater aber keineswegs durch den Holocaust verloren hat, sondern durch eine - in seiner Wahrnehmung - USamerikanische Phase der "Tyrannei“, gehört ebenfalls zu den „ironic reversals“ (Gross 1980: 53) des Films: Babes Vater, ein Historiker, war in der McCarthyÄra als Kommunist gebrandmarkt worden, hatte seinen Posten als Universitätsprofessor verloren und sich daraufhin das Leben genommen. Dieses Kindheitstrauma überwindet Babe nun, indem er seinen Rachewunsch auf Szell projiziert. Zwar ist seine Verfolgung des Nazi-Arztes auch durch dessen Mord an seinem Bruder und die durch ihn erlittene Folter motiviert, die Entschlossenheit aber gewinnt Babe aus dem Verlust seines Vaters. Er stellt Szell mit jener Waffe, die sein Vater dazu verwendet hatte, sich das Leben zu nehmen. Das Paradox, das durch diese Übertragung entsteht, formuliert Gross wie folgt: "since his anger is in a sense misplaced, stemming in reality from his father's death, the Nazi becomes the Jew's scapegoat." (1980: 64) Dass Babe die Verfolgung Szells als "therapeutic experience“ (Gross 1980, 62) erlebt und sich durch dessen Tod von seinem Kindheitstrauma befreit, macht die Schlussszene des Films deutlich, in der er die bisher sorgsam gehütete Waffe seines Vaters im

19 Auch zwischen den Gegenspielern Babe und Szell gibt es irritierende Effekte der Spiegelung: Beide haben einen Bruder, der ums Leben kommt (Szells Bruder stirbt zu Beginn des Films in einem grotesken Autorennen mit einem alten Juden, Scylla wird von Szell ermordet). 
Wasser versenkt. Sein Ende findet Szell gleich zweifach nach dem Prinzip des ius talionis, der spiegelnden Strafe, indem Babe ihn zunächst in deutscher Sprache zwingt, seine eigenen Diamanten zu schlucken („Essen!“), und Szell dann bei dem Versuch, seine Diamanten zu retten, in seinen eigenen Dolch stürzt.

Wie in The Boys from Brazil konzentriert sich die Handlung auch in Marathon Man auf die USA. Szell wird internationalisiert. Weder ist sein Name deutsch (das wäre allenfalls Schell), noch hat er einen deutschen Akzent. Er äußert sich nie politisch und zeigt keine Nazi-Symbole, es geht ihm ausschließlich um Geld. Noch überraschender ist die dargestellte Kollaboration zwischen dem US-Geheimdienst und dem NS-Kriegsverbrechter, werden Nationalsozialisten als klassische villains US-amerikanischer Filme doch sonst in der Regel möglichst weit von ihren US-amerikanischen Gegnern distanziert. Die Komplizenschaft zwischen der Figur, die paradigmatisch das Böse verkörpert, und den US-Geheimagenten rückt diese in eine kritische Perspektive und bringt ein grundlegendes Misstrauen gegenüber der Regierung und dem Geheimdienst zum Ausdruck, so dass der KZ-Arzt hier nicht zuletzt der Verhandlung eines inneramerikanischen Vertrauensverlusts dient. (Dieser resultiert, so lässt sich vermuten, aus den Konflikten der McCarthy-Zeit, aus dem Vietnam-Krieg, aber auch aus dem noch nicht lange zurückliegenden Watergate-Skandal). Das Verbrechen wird adaptiert und adoptiert. Die Bedrohung geht von den Vertretern des eigenen Staates aus. Manichäische Figuren-Konstellationen, erwartbare Charakterisierungen ${ }^{20}$ und klassische PlotElemente werden unterlaufen, so dass eine Verunsicherung darüber entsteht, ob und wie ,Gut' und ,Böse' voneinander abgrenzbar sind.

Der Film Nichts als die Wahrheit (1999) schließlich entwirft ein kontrafaktisches Szenario in Deutschland. Er stellt die Frage: Was wäre, wenn Mengele sich in der Gegenwart vor einem deutschen Gericht verantworten würde? Der Film inszeniert die unheimliche Heimkehr des Täters, "the uncanny return of Mengele in a realist mode" (Bangert 2014: 63). Das Auftauchen des totgeglaubten KZ-Arztes bezeichnet der Staatsanwalt als "Alptraum", was eine psychoanalytische Deutung nahelegt: die Wiederkehr des Verdrängten. ${ }^{21}$ Mengele stellt sich im Film den deutschen Behörden, um

20 Auch dass in Marathon Man judenfeindliche Klischees auf den Nazi-Arzt übertragen werden (Szell ist geldgierig; er handelt mit Diamanten; er steuert ein internationales Netzwerk gleichsam als kriminelle ,Weltverschwörung'), trägt zu der Destabilisierung klischeehafter (und in diesem Fall: rassistischer) Vorstellungen bei. Irritierend wirkt, dass nicht nur antisemitische Vorstellungen auf den Nazi-Arzt übertragen werden, sondern er auch äußerlich, durch das Rasieren seines Kopfes, an die Behandlung von KZ-Häftlingen erinnert (vgl. Gross 1980, 58). Eine ähnliche paradoxe Übertragung findet in dem Film My Father statt, in dem Mengele bei seinem ersten Auftritt unter der Dusche zu sehen ist, eine Einstellung, welche den Massenmord in den Gaskammern aufruft.

${ }^{21}$ Zur mangelnden Aufarbeitung der Verbrechen der NS-Medizin nach 1945 vgl. Freimüller (2003). Als Belastung für die deutsch-jüdischen Beziehungen inszeniert der Film den 
„seine Wahrheit“ kundzutun, derzufolge er in Auschwitz seiner Pflicht als Arzt gerecht geworden sei, indem er aus Menschlichkeit getötet habe.

Der junge Anwalt und Mengele-Forscher Peter Rohm wird im Film mit der Aufgabe betraut, Mengele vor Gericht zu verteidigen. Von dessen Helfershelfern entführt, trifft er ihn in seinem Exil und wird mit einem Dilemma konfrontiert: Der sterbenskranke Mengele ist bereit, sich einem deutschen Gericht zu stellen, unter der Voraussetzung, dass Rohm seine Verteidigung übernimmt. Überzeugt vom deutschen Rechtssystem, das jedem Angeklagten einen Verteidiger garantiert, und besessen von der Frage nach der Motivation für Mengeles Taten, willigt Rohm schließlich ein, obwohl er von der Schuld seines Mandanten überzeugt und von dessen Verbrechen abgestoßen ist. Rohm entwickelt eine doppelte Verteidigungsstrategie, indem er Mengele einerseits als „Kind seiner Zeit" charakterisiert und die Popularität der Eugenik im medizinischen Diskurs der 1920er und 30er Jahre hervorhebt - zum Beispiel durch den Hinweis auf den Text Die Freigabe der Vernichtung lebensunwerten Lebens (1920) von Alfred Hoche und Karl Binding; und indem er die Verbrechen andererseits als Euthanasie, als Akt der Gnade und Erlösung von den Qualen im Konzentrationslager und der bevorstehenden Vergasung deutet. Diese Rechtfertigung seiner Taten vertritt auch Mengele selbst: „Ich habe Auschwitz nicht erfunden. Ich wollte helfen.“ Und er ergänzt: „Die besten Ärzte töten.“ Bewusst stellt Rohm Mengeles Morde als lediglich graduelle Steigerung zeitgenössisch diskutierter - und beispielsweise in der Schweiz legaler - Formen der Sterbehilfe dar. ${ }^{22}$

Diese Verteidigung erweist sich als so schwer widerlegbar, dass Rohm beginnt, seinen eigenen Erfolg $\mathrm{zu}$ fürchten. Im letzten Teil seines Schlussplädoyers handelt er daher seiner Rolle als Verteidiger zuwider, indem er Mengeles Taten plötzlich als unentschuldbare Verbrechen charakterisiert und einen Schuldspruch für seinen Mandanten fordert. Nur durch den Regelbruch des Anwalts kann das Scheitern des Rechtsstaates - der, wie die

Gerichtsprozess, indem er die Entzweiung zwischen Rohm und seiner Frau schildert, die den jüdischen Namen Rebekka trägt. Sie wirft ihm vor, zu Mengeles Komplizen geworden zu sein, und wendet sich von ihm ab; erst Rohms Plädoyer für eine Verurteilung Mengeles leitet die wie der Streit symbolisch lesbare - Versöhnung ein, aus der dann ein gemeinsames Kind hervorgeht (vgl. Steinle 2013: 171-172): eine deutsch-jüdische ,Symbiose'.

${ }^{22}$ Auf diese Weise liefert der Film implizit auch einen Beitrag zur zeitgenössischen Debatte um die Sterbehilfe und ihre Legalisierung (vgl. z.B. Grimm/Hillebrand 2009). Zur Rolle der nationalsozialistischen ,Euthanasie ${ }^{\prime}$ in der aktuellen Sterbehilfe-Debatte und für juristische Entscheidungen vgl. Benzenhöfer (2000), Burleigh (2000), Schmuhl (2000), Zimmermann-Acklin (2000). Mengele selbst, der im Film an fortgeschrittenem Knochenkrebs leidet, wünscht sich jedoch keineswegs den Tod. Als ihn einer seiner ehemaligen Helfer mit einer als „Vitaminspritze“ getarnten Injektion töten möchte, weiß er dies zu verhindern. Hier - wie auch in Mengeles physiologischer Untersuchung und Vermessung durch das Gericht zur Feststellung seiner Identität - kommt es zu einer Verkehrung: Mengele wird in Situationen dargestellt, die an den Umgang der Nationalsozialisten mit ihren Opfern erinnern. 
Richterin betont, mit diesem Verfahren auf dem Prüfstand stehe - an der Verurteilung eines nationalsozialistischen Massenmörders verhindert werden. Das Urteil selbst aber spart der Film aus und lässt so letztendlich offen, wie das Gericht Mengeles Taten beurteilt. Allein die Möglichkeit eines Freispruchs, die durch Rohms engagierte, wenn auch widerwillige Verteidigung eine Zeitlang denkbar erscheint, lässt die deutsche Justiz jedoch ambivalent erscheinen - so ambivalent wie den Namen des Anwalts, der zwischen dem römischen Recht Rom - und dem Vertreter der Nazi-Verbrechen - Röhm - changiert.

Das Urteil, so scheint es, bleibt letztlich dem Publikum überlassen, das damit nicht nur zu einer Auseinandersetzung mit der bundesdeutschen Rechtsprechung, sondern auch mit dem eigenen Verhältnis zum Verbrechen und zum Verbrecher herausgefordert wird. Rohm wird zum "devil's advocat" (Bangert 2014: 68). Indem er Mengele verteidigt, leistet er eine Überzeugungsarbeit, der nicht nur das Gericht, sondern auch die ZuschauerInnen des Films ausgesetzt sind. Der Film inszeniert so ein "seductive encounter with Mengele" (Bangert 2014: 65):23 "Nichts als die Wahrheit thus adopts the crime thriller scenario of a mind game in which the investigator, through his occupation with the nature of evil, runs the risk of being absorbed by it." (Bangert 2014: 68) Durch seine "transgressive strategy of seducing the viewer into momentarily assuming Mengele's subjective perspective“ (Bangert 2014: 66) rückt der Film den flüchtigen Nazi-Verbrecher, 20 Jahre nach dessen Tod, in eine bedrohliche Nähe zur Gegenwart (vgl. auch Loewy 2004: 98). Er zeigt auf, dass nicht nur jede/r zum Täter werden kann, sondern dass dem nationalsozialistischen Verbrecher noch immer das Potential einer Verführung zum Bösen innewohnt.

$\mathrm{Zu}$ dieser verführerischen Wirkung trägt das Charisma der Mengele-Figur (gespielt von Götz George) bei: Ihre Souveränität und Gelassenheit ebenso wie ihre körperliche Präsenz und ihr „piercing gaze“ (Bangert 2014: 66) sowie ihre dirigierenden Gesten machen sie zu einem Objekt bzw. Subjekt der Faszination. Fokussiert wird diese Ausstrahlungskraft durch wiederkehrende close-ups auf Mengeles Gesicht oder Lippen, was zugleich eine unheimliche Intimität mit dem Täter erzeugt. Mengele wird so "an object of attraction and repulsion“ (Bangert 2014: 57).

Das letzte Wort überlässt der Film Mengele, der weiterhin auf seiner Unschuld beharrt und ein eigenes Verteidigungsplädoyer hält, das kein Ende $\mathrm{zu}$ finden scheint. Dem Publikum rückt er in dieser Szene auf dreifache Art nahe: (1.) Sein Gesicht - und schließlich dessen einzelne Partien - füllen den ganzen Bildschirm; (2.) seine Ansprache dauert bis in den Abspann hinein (der Beginn der credits wird eingeblendet, während Mengele aus dem Off weiterspricht, dann taucht sein Gesicht ein weiteres Mal auf), und sie reicht damit über die Diegese und die fiktionale Welt hinaus; (3.) im letzten Satz des

${ }^{23}$ Vgl. auch die Bilanz Rohms: „Dieser Prozess hat gezeigt, wie verführbar wir alle sind, wie klein der Schritt zum Verlust all unserer menschlichen Werte ist.“ 
Films spricht Mengele metaleptisch das Publikum an und scheint dessen Identifikation einzufordern: „Sehen Sie wenigstens ein bisschen von sich selbst in mir?" Statt eine Verurteilung zu präsentieren, fordert der Film die ZuschauerInnen dazu auf, den Blick auf sich selbst zu richten: Am Ende steht also keineswegs ,nichts als die Wahrheit', sondern eine offene Frage und ein ,Auftrag' - formuliert durch die Figur Mengele selbst.

Dieser filmrhetorischen Annäherung an den Täter entspricht seine Darstellung als keineswegs fundamental ,Anderer' - weder der Figuren im Film noch der ZuschauerInnen. Der Film betont seine Normalität. Der Anwalt Peter Rohm stammt aus Mengeles Heimatstadt Günzburg. Er arbeitet an einem Buch mit dem Titel Mengele - Einer von uns. In einem Gespräch mit dem Staatsanwalt betont er, es sei keineswegs davon auszugehen, dass, wir die besseren Menschen seien. Einen ähnlichen Effekt der Normalisierung des Verbrechers hat die Aufdeckung der Schuld vermeintlich unschuldiger Figuren: Rohm findet erst durch einen Hinweis von Mengele heraus, dass auch seine Mutter - allerdings unwissentlich - im Rahmen der T4-Aktion Morde begangen hat, dass also auch seine nächste Angehörige während der NS-Zeit schuldig geworden ist.

Während im Film und vor dem Bildschirm einerseits eine Annäherung an die Nachgeborenen stattfindet, welche die Anomalität Mengeles relativiert, inszeniert Nichts als die Wahrheit Mengele andererseits und im Widerspruch dazu als psychopathologischen Täter. Der Film stattet die Figur mit einer "monstrous physique“ (Bangert 2014: 67) aus, die eine „voyeuristic fascination with his corporeality“ (Bangert 2014: 67) auslöst. Seine bleiche Haut und seine spitzen Fingernägel (vgl. 1:28) rufen Vampir-Figuren wie Nosferatu oder Dracula in Erinnerung; und trotz seiner körperlichen Hinfälligkeit wirkt Mengele machtvoll und einflussreich (so findet die Gerichtsverhandlung auf seine Initiative statt, und bis zu den Schlussplädoyers gelingt es ihm, den Lauf der Ereignisse in seinem Interesse zu lenken). Zudem erinnert die Figur in ihrer visuellen Inszenierung an Hannibal Lecter, den Protagonisten des Films Silence of the Lambs (USA 1991, Regie: Jonathan Demme), einen kannibalischen Serienkiller (Bangert 2014: 65). Der Mengele-Figur wird so eine Reihe von außergewöhnlichen Attributen zugeschrieben: eine "seductive but manipulative intelligence, repulsive yet intriguing monstrosity, as well as extreme body horror" (Bangert 2014: 65). Durch den Schauspieler Götz George wird die Figur außerdem mit Fritz Haarmann assoziiert, einem Serienmörder, den George im Film Der Totmacher (1995, Regie: Romuald Karmakar) verkörperte. Haarmann tötete seine Opfer unter anderem mit einem Biss in den Kehlkopf beim Sexualakt, woraufhin er die Leichen zerlegte, und ist deswegen auch als Vampir, Kannibale und Monster ins kollektive Gedächtnis 
eingegangen. ${ }^{24}$ Beide Assoziationen rücken Mengele in die Nähe psychopathologischer Verbrecher, eine Deutung, die auch der Staatsanwalt in seinem Schlussplädoyer nahelegt, wenn er ihn als "Psychopathen“ bezeichnet und ihm ein "krankes Ich“ zuschreibt. Die Täterinszenierung in Nichts als die Wahrheit bleibt so ambivalent. Sie vereint zwei widersprüchliche Täterbilder: auf der einen Seite die Vorstellung vom ,ordinary man',25 der sich nicht grundlegend vom Rest der Bevölkerung unterscheidet; auf der anderen Seite das diabolische Monster und der pathologische Kriminelle ${ }^{26}$, (Denk-)Figuren, die einer "Distanzgewinnung“ (Paul 2002: 16) dienen und den Täter als ,Anderen' aus der Durchschnittsbevölkerung ausschließen.

\section{NACHFAHREN}

Die Figur des KZ-Arztes wird in verschiedenen Filmen mit Figuren der Söhneund Töchter-Generation konfrontiert: sowohl mit eigenen Nachkommen als auch mit Nachfahren der Opfer, wobei letztere in der Regel als VerfolgerInnen auftreten. Der überlebende, reuelose Täter bietet sich als Objekt einer persönlichen Zwiesprache zwischen den Generationen beispielhaft an. Die literarische Auseinandersetzung mit den Nazi-Tätern aus der Perspektive der nachfolgenden Generation wird als „Väterliteratur" bezeichnet (Mauelshagen 1995; Reidy 2012, 2015). Auf Josef Mengele aus Sicht der Söhne bzw. Töchter konzentrierten sich Peter Schneider in der Erzählung Vati (1987) und Lucía Puenzo im Roman Wakolda (2011). ${ }^{27}$

In der Verfilmung von Vati, der englischsprachigen Produktion My Father, Rua Alguem 555 (Italien 2003), besucht Mengeles erwachsener Sohn seinen Vater in Brasilien. Bereits die Besetzungspolitik bestimmt die Semantik des Films. Den flüchtigen Nazi und Massenmörder Josef Mengele spielt Charlton Heston, ausgerechnet ein Schauspieler, der durch Ben Hur (1959) in der Rolle eines

\footnotetext{
${ }^{24}$ Vgl. den 1925 erstmals in der Reihe Außenseiter der Gesellschaft - Verbrechen der Gegenwart erschienenen Bericht Haarmann. Die Geschichte eines Werwolfs von Theodor Lessing, aber auch jüngere literarische Bearbeitungen (Steinhauer 2017) und journalistische Berichte (vgl. Müller 2008).

${ }^{25}$ Den Begriff ,ordinary men' führte Henry V. Dicks (1972) in die Täterforschung ein, nachdem bereits Herbert Jäger (1982 [1967]) den Anstoß für eine Neufokussierung auf die ,gewöhnlichen Verbrecher' geliefert hatte (vgl. Paul 2002: 33-35). In der Folge wird er zu einem Zentralbegriff in der Diskussion über die Einordnung der Täter, vgl. Browning (1992), Matthäus (1996), Westermann (1998).

${ }^{26}$ Während die Betrachtung der Täter als ,ordinary men' erst Ende der 60er Jahre einsetzte, dominierte die frühe Täterforschung das Bild vom Täter als pathologischem Kriminellen und diabolischem Monster, vgl. Paul (2002: 16-20).

${ }^{27}$ Bei der Hauptfigur in Puenzos Roman handelt es sich, strenggenommen, weder um eine Verwandte des KZ-Arztes noch um die Tochter eines seiner Opfer oder eine Verfolgerin. Da der Roman das Verhältnis zwischen Mengele und einer Vertreterin der nachfolgenden Generation thematisiert, wird der Film dennoch in diesem Abschnitt behandelt.
} 
jüdischen Opfers und Helden bekannt wurde. In Kontrast zu dieser StarBesetzung bezeichnet der jüdische Anwalt Mengele dann als „a common, even banal man“. Mengeles Sohn Rolf spielt Thomas Kretschmann. Auch diese Besetzung ist, ohne dass der deutsche Schauspieler dafür verantwortlich zu machen wäre, problematisch. Denn die beiden blonden, gutaussehenden, sportlichen Männer, Vater und Sohn, scheinen Mengeles Phantasien von der Genealogie der arischen Rasse naiv zu bestätigen.

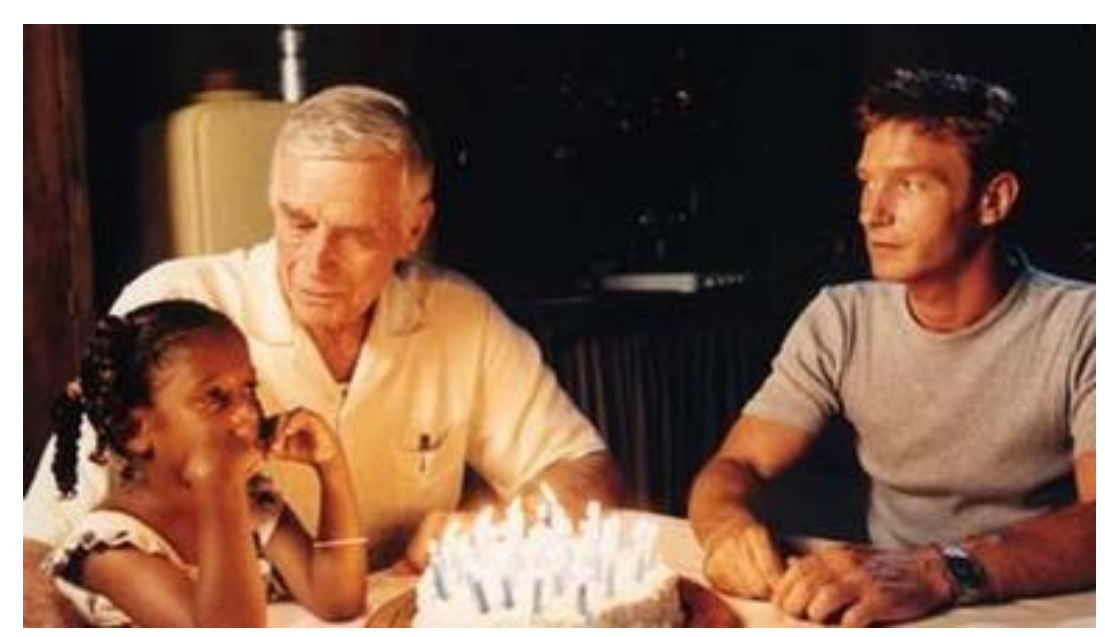

Abbildung 7: Josef und Rolf Mengele (Charlton Heston und Thomas Kretschmann) mit einem brasilianischen Mädchen in My Father, Rua Alguem 555 (Italien 2003).

Die Figur des Juden (F. Murray Abraham als Paul Minsky) ist ebenso stereotyp angelegt: als dunkelhaariger Anwalt mit auffallender Nase. Mengeles Ideologie scheint die Inszenierung des Films zu bestimmen. Eingespielt wird klassische Musik: „Die Moldau“, Brahms. Diese extradiegetische Filmmusik bildet den Soundtrack des gebildeten Täters. Unter die Bilder des Films werden dagegen Schwarzweiß-Aufnahmen aus Konzentrationslagern montiert. Diese Visionen von Auschwitz entsprechen der Phantasie des jungen Protagonisten: Der Sohn ist sekundär traumatisiert.

Der Film enthält merkwürdige Unstimmigkeiten: Öffentliche Demonstrationen gegen den flüchtigen Nazi hat es in Brasilien nicht gegeben. Die Ausgrenzung der Mengeles in Deutschland, v.a. des Jungen in der Schule, ist unglaubwürdig; als seien die Deutschen kurz nach dem Krieg Antifaschisten gewesen, die sich gegen die Nazis und sogar deren Nachkommen stellten. Weitere Unstimmigkeiten liegen in der Aussprache. Der Vater (Heston als Mengele sr.) hat keinen deutschen Akzent, der Sohn (Kretschmann als Mengele jr.) hingegen schon. Der Sohn nennt seinen Vater „Papa“, und nicht, wie im Titel von Peter Schneiders Erzählung, provokativ zärtlich „Vati“. Der 
Titel des Films lautet entsprechend nicht etwas „Daddy", sondern "My Father". Die Pointe der kindlichen Überspitzung geht damit verloren. ${ }^{28}$

Auch in der Verfilmung von Wakolda (Argentinien 2013) wird die Perspektive eines Kindes eingenommen, in diesem Fall die des 12jährigen argentinischen Mädchens Lilith, die zu dem geheimnisvollen deutschen Arzt eine eigenartige Zuneigung entwickelt. Mengele lebt im Gasthaus ihrer Familie und behandelt Lilith wegen ihrer Wachstumsschwierigkeiten.

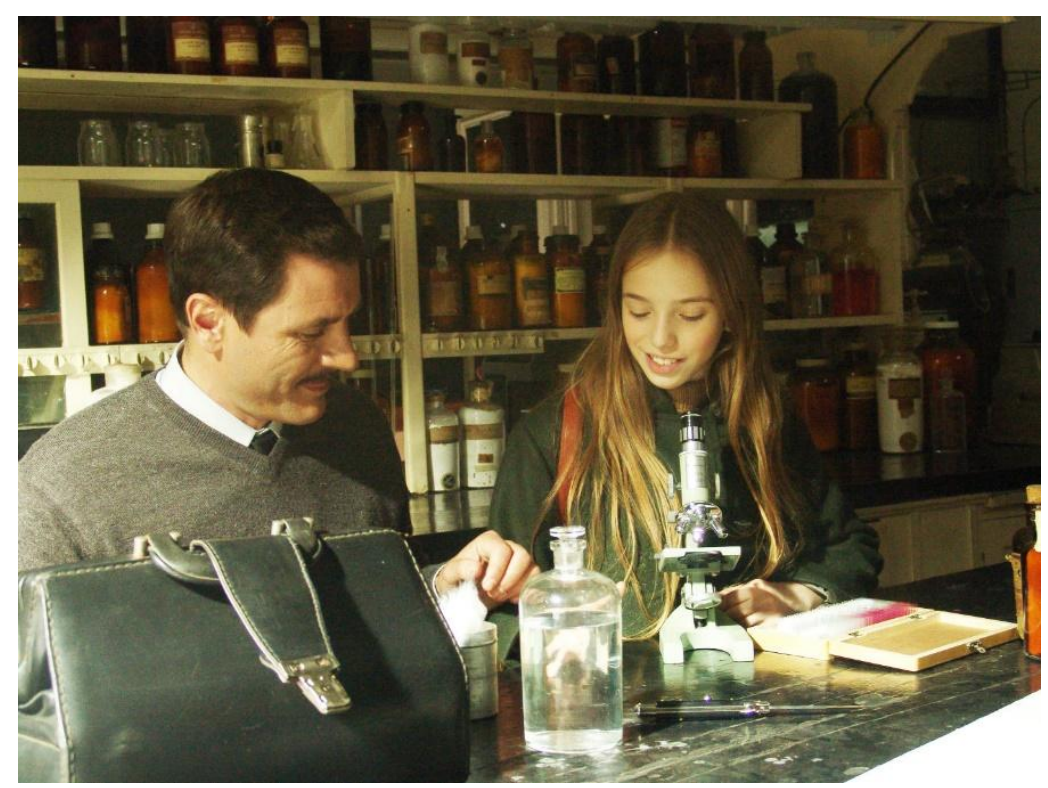

Abbildung 8: Geheimnisvolle Sympathie - Mengele (Alex Brendemühl) und Lilith (Florencia Bado) in Wakolda (Argentinien 2013).

Das Leitmotiv in Lucía Puenzos Roman ebenso wie im Film ist das der Puppen. Sie verkörpern Mengeles Wunsch, einen makellosen Körper hervorzubringen, der sich am rassistischen Ideal genetischer ,Reinheit" orientiert. Während die ,unvollkommene' Lilith zu seinem Forschungsobjekt und zu seinem Opfer wird, tritt Mengele bei der Puppenherstellung ihres Vaters als Investor und Mitschöpfer auf. Als tote Figuren erinnern die Puppen zudem an die Leichen von Auschwitz, als massenhaft hergestellte an die fabrikmäßige Tötung und als künstlerisches Bild an E. T. A. Hoffmanns Novelle „Der Sandmann“ (2000 [1816]) - und damit an Sigmund Freuds berühmte Deutung dieses Textes, „Das Unheimliche“ (1999 [1919]). Was wir als unheimlich empfinden, so Freuds These, ist uns eigentlich allzu vertraut. Es ist die Wiederkehr des Verdrängten. Die argentinische Gesellschaft der 1950er und 60er Jahre, so ließe sich diese Assoziation auf den Film beziehen, ist geprägt von einer Verdrängung der faschistischen Verbrechen, deren Täter hier wieder zum

\footnotetext{
${ }_{28}^{2}$ Das Drehbuch, an dem Peter Schneider anfänglich mitwirkte und das dessen Kommentare zeigt, liegt im Archiv in der Akademie der Künste, Berlin.
} 
Vorschein kommen. Deutsche Kriegsverbrecher können sich ungehindert im Land bewegen und der juristischen Verfolgung entziehen.

Mengele erscheint im Film zunächst in einem neutralen oder gar positiven Licht: Wiederholt zeigen Großaufnahmen seine handschriftlichen Aufzeichnungen und Zeichnungen: Sie ästhetisieren den Verbrecher - geradezu leonardesk als Universalgelehrten, er erscheint als Wissenschaftler und Künstler. Die Wachstumsbehandlung, die er an Lilith durchführt, wird von ihr und ihrer Mutter befürwortet (Lilith leidet in der Schule unter Hänseleien wegen ihrer geringen Körpergröße). Sie erinnert im argentinischen Kontext zudem an den Fußballstar Lionel Messi, eine nationale Ikone, der in seiner Jugend unter einer Wachstumsstörung litt, die mit Hormonen behandelt wurde. Sie scheint vor diesem Hintergrund legitimiert zu sein. Erst im Laufe des Films wandeln sich die Charakterisierung und die Bewertung der Figur: Lilith beginnt zunehmend unter der Hormonbehandlung zu leiden. Mengeles Skrupellosigkeit wird schließlich deutlich, als Liliths Mutter Zwillinge bekommt, die aufgrund einer Frühgeburt in Lebensgefahr schweben. Mengele, der Zwillingsforscher, entscheidet sich, nur dem schwächeren der beiden Säuglinge Medikamente zu geben, und macht die Kinder und ihre Familie damit rücksichtslos zu seinen Versuchsobjekten.

Der Abspann von Wakolda enthält zwei Sonderbarkeiten: Mengele wird als „eterno fugitivo“, als „ewiger Flüchtling“ bezeichnet, d.h. der geflohene Nazi wird gleichsam zum ewigen Juden, Ahasver. (So wie er in My Father mit Ben Hur assoziiert wurde.) Und sein Tod wird in Zweifel gezogen. Nur „según la versión oficial“, „nach öffentlicher Darstellung“, sei Mengele ertrunken. Damit wird, einmal mehr, der Horror-Topos bedient von dem Bösen, das überwunden zu sein scheint, aber doch überlebt.

Der israelische Film The Debt (Israel 2007, Originaltitel: החוב) und sein gleichnamiges US-amerikanisches Remake (USA 2010) rücken die VerfolgerInnen in den Fokus. (Die US-amerikanische Version folgt im Wesentlichen der Handlung des Vorgängerfilms und nimmt Änderungen lediglich im Detail vor.) Im Mittelpunkt der Handlung steht die MossadAgentin Rachel, die gemeinsam mit zwei männlichen Kollegen 1964 den fiktiven ,Arzt von Birkenau', Max Rainer (Edgar Selge) ${ }^{29}$, in Ost-Berlin aufspürt und entführt. Die geheime Überführung nach Israel scheitert jedoch, laut Aussage der anderen Agenten erschießt Rachel den Deutschen bei einem Fluchtversuch. In Israel werden die drei daraufhin als (National-)Helden gefeiert. In der Erzählgegenwart Ende der 1990er Jahre stellt sich jedoch heraus, dass der Nazi-Arzt entkommen konnte, die Heldenlegende der drei AgentInnen also auf einer Lüge beruht. (Eine Narbe im Gesicht, die Rachel aus dem Kampf mit Rainer davontrug, wird in diesem Zusammenhang als Stigma lesbar.) Die

${ }^{29}$ Im US-amerikanischen Remake heißt die Figur Dieter Vogel und wird gespielt von Jesper Christensen. 
Lüge droht aufzufliegen, als ein Bewohner eines Seniorenheims in der Ukraine behauptet, der ,Arzt von Birkenau' zu sein. Um ihren Ruf zu schützen, macht sich die inzwischen etwa 60jährige Rachel auf den Weg nach Kiew, um den ehemaligen KZ-Arzt nun doch noch ums Leben $\mathrm{zu}$ bringen. Die finale Konfrontation überleben weder sie noch ihr Gegenspieler: Sie töten sich gegenseitig.

Wie in Nichts als die Wahrheit steht auch in The Debt die Frage nach dem Umgang mit dem Täter im Vordergrund, anders als im deutschen Film geht es jedoch nicht um die juristische Aufarbeitung, sondern problematisiert wird das Vorgehen des israelischen Geheimdienstes. Inspiriert von der Entführung Adolf Eichmanns aus Buenos Aires durch den Mossad im Mai 1960, entwirft der Film ein fiktives Szenario, in dem die AgentInnen gleich mehrfach scheitern. Es gelingt ihnen nicht, ihren Gefangenen außer Landes zu bringen - ein Versagen, das sie mit einer Lüge vertuschen. Statt dem grausamen Täter moralisch eindeutige Verfolgerfiguren gegenüberzustellen, lässt der Film die AgentInnen selbst moralisch fragwürdig werden. Von Beginn an wirken die drei im Umgang mit ihrer „Mission“ nicht souverän. Besonders deutlich wird dies an der Protagonistin Rachel, die, um den nunmehr als Gynäkologe praktizierenden Rainer auszuspionieren, zu seiner Patientin wird. Dreimal sucht sie ihn - ganz unnötigerweise - in seiner Praxis auf und lässt sich von ihm behandeln. Die intime Entblößung vor dem ehemaligen KZ-Arzt und die passive Situation als Patientin sind für sie, wie an ihrem Gesicht abzulesen ist, schwer zu ertragen. Der unerfüllte Kinderwunsch als Vorwand für die Behandlung ruft die von den Nationalsozialisten praktizierten Zwangssterilisationen und die Experimente zur Erhöhung der Fruchtbarkeit in Erinnerung - dies umso mehr, da Rachel berichtet, ihre Mutter habe nach dem Krieg keine Kinder mehr bekommen können. Indem Rachel sich freiwillig in die Hand des Nazi-Arztes begibt, wird diese Opferrolle fortgesetzt. Auch und gerade während ihrer Verfolgung des Nazi-Verbrechers bleiben die AgentInnen im Film von der Erfahrung des Holocaust bestimmt. Sie sind - wie in My Father der Sohn des Täters - (sekundär) traumatisiert. ${ }^{30}$ Ihr Leiden ist mit dem Ende des Zweiten Weltkrieges nicht abgeschlossen.

Die Szenen beim Frauenarzt sind als Intimisierung der Beziehung zum Täter zu lesen, der zugleich symbolisch zum medizinischen ,Vater ${ }^{`}$ des Kindes wird, mit dem Rachel in dieser Zeit (von einem ihrer beiden männlichen Kollegen) schwanger wird. So steht auch die nächste Generation weiterhin in einer allzu engen Beziehung zu den Tätern, die Beziehung zwischen Agentin und Täter verliert ihre Eindeutigkeit. Eine allzu große Nähe zum Täter stellt sich des weiteren ein, als die drei AgentInnen den ehemaligen Nazi-Arzt in

${ }^{30}$ Mindestens zwei der AgentInnen sind sekundär traumatisiert: Zvis Familie wurde in Auschwitz ermordet; angedeutet wird, dass Rachels Mutter möglicherweise sterilisiert wurde. Der Leidensdruck, unter dem die AgentInnen stehen, wird daran deutlich, dass Rachel immer wieder weinend gezeigt wird, ohne dass jeweils ein Anlass auszumachen wäre. 
einer kleinen Wohnung gefangen halten, während sie auf Anweisungen ihrer Vorgesetzten warten. Obwohl einer der männlichen Agenten alle Gespräche mit dem Täter verbietet und die Distanz mit allen Mitteln aufrecht erhalten will („He's not a human being“), stellen sich zwangsläufig Nähesituationen ein, welche die AgentInnen zwingen, mit ihrem Gefangenen zu interagieren. Rachel füttert und rasiert ihn und malt ihm seinen Gerichtsprozess aus, Ehud zieht sich vor seinen Augen aus und scheint in einer hilflosen Geste durch das Vollführen von Liegestützen seine physische Überlegenheit demonstrieren $\mathrm{zu}$ wollen. Während der Zeit, welche die AgentInnen mit dem Nazi in der Wohnung verbringen, werden sie immer nervöser, reizbarer, unprofessioneller, und sie zerstreiten sich über das weitere Vorgehen, so dass hier das Bild entsteht, dass die VertreterInnen der Opfer mit ihrer neuen Rolle als VerfolgerInnen überfordert sind. Die Verkehrung der Rollen - der Täter ist nun ein Gefangener, die Juden sind seine Bewacher - bedeutet für die AgentInnen ein moralisches Dilemma: Sie stehen vor der Schwierigkeit, den Täter zu verfolgen, ohne selbst zu Tätern zu werden. Dieses Dilemma illustriert der Film, indem sich Rachel dem KZ-Arzt zweimal angleicht: Vor der Entführung betäubt sie ihn in seiner Praxis mit einer Spritze, sie nutzt also ihrerseits medizinische Utensilien für eine nicht-medizinische Handlung, die gegen den Willen des Betroffenen durchgeführt wird. Als sie Rainer im ukrainischen Seniorenheim verfolgt, verkleidet sie sich als Krankenschwester, sie nutzt also den ,Deckmantel' der Medizin für einen geplanten Mord. Auch wenn ihr Vorgehen mit den Verbrechen des KZ-Arztes nicht gleichzusetzen ist, rückt der Film das Handeln der Agentin motivisch in eine moralische Ambivalenz und wirft zumindest die Frage auf, ob hier ein Rollenwechsel stattfindet, der ihr Verhalten als Vertreterin Israels delegitimiert.

Mehrfach betont Rachel, sie wolle nicht töten, „not even him“. Sie gerät dann aber in eine Situation, die sie zur Mörderin (so bezeichnet Rainer sie) macht. Im Badezimmer des Seniorenheims kommt es zur finalen Konfrontation zwischen dem Nazi und der Nazi-Jägerin, bei der sie Rainers Kehle mit einer Spiegelscherbe durchschneidet und dabei ihrerseits von ihm tödlich verletzt wird. Die Mordwaffe lässt zwei Deutungen der Tat zu: Einerseits erscheint Rainers Tötung durch Rachel als Rache, hier sogar ganz buchstäblich als "Spiegelstrafe“, abermals nach dem Prinzip des ius talionis (wie in Marathon Man), um so mehr, da Rainer die Scherbe aus dem Spiegel selbst herausbrach, als er seine Gegnerin mit dem Kopf dagegen stieß. Andererseits ist der Spiegel ein Medium der Selbstreflexion, des gebrochenen Selbstbildes, das bei Rachel jenes einer Frau ist, die getötet hat, obwohl sie nie töten wollte. Hinzu kommt, dass ihre Motivation für diesen Mord moralisch fragwürdig ist: 1997 ist sie nicht mehr israelische Geheimagentin, die einen Verbrecher verfolgt, sondern 
sie handelt als Privatperson und aus Eigennutz, damit ihre Lüge nicht aufgedeckt wird und das öffentliche Bild der Heldin intakt bleibt. ${ }^{31}$

Die Filme problematisieren den Umgang mit dem Täter, indem sie die Nachfahren (sowohl Mengeles Sohn als auch die jüdischen Nachkommen der Opfer) an einer moralisch eindeutigen Positionierung scheitern lassen. Dem Protagonisten von My Father gelingt die Abgrenzung von Mengele nicht, er führt ihn keiner Bestrafung zu; und die israelischen AgentInnen lassen den Täter entkommen und machen sich der Lüge sowie des Mordes aus egoistischen Motiven schuldig. In Wakolda steht die 12jährige Lilith für eine unbedachte und problematische Nähe zum Täter, während sie gleichzeitig zu dessen Opfer wird.

\section{Horror, ACTION, Trash}

Neben den Holocaust-Filmen, den Darstellungen des entkommenen Täters und den Dramen der Generationen hat sich die Figur des KZ-Arztes immer weiter verselbständigt, bis sie in diversen Genres als Chiffre des Bösen fast beliebig einsetzbar wurde: im Horror (In A Glass Cage, 1986; The Unborn, 2009; The Human Centipede, 2009), im Trash (Surf Nazis Must Die, 1987) oder im Superhelden-Film (X-Men, 2011; Captain America, 2011) und nicht zuletzt auch im Sexploitation- und Pornofilm (z.B. Ilsa, She Wolf of the SS, 1974; Dr. Mengele, 2005).

Ein Beispiel soll an dieser Stelle genügen: Der Horrorfilm The Unborn (USA 2009) assoziiert die Figur Josef Mengele (gespielt von Braden Moran) mit einem übernatürlichen Bösen. Die junge US-Amerikanerin Casey wird in Träumen und Visionen von einem Geist heimgesucht. Die Erklärung für diese unheimlichen Vorgänge findet Casey in "a suppressed family inheritance" (Langford 2013: 120). Sie findet heraus, dass ihre Großmutter und deren Zwillingsbruder in Auschwitz von Mengele für Experimente missbraucht wurden. Als der Junge während eines dieser Experimente stirbt, ergreift ein Dybbuk Besitz von seinem Körper. ${ }^{32}$ Auf diese Weise in die Welt gebracht, sucht der Geist auch die nachfolgenden Generationen der Familie heim: Caseys Zwillingsbruder starb auf Wirken des Geistes bereits im Mutterleib; ihre Mutter hat sich, verfolgt vom Dybbuk und aus Angst um das Leben ihrer Tochter, das Leben genommen. Als der Dybbuk immer stärker wird, unterschiedliche Gestalten annimmt und mehrere Menschen in Caseys Umfeld tötet, sucht sie Hilfe bei einem Rabbi, der sich schließlich bereit erklärt, einen jüdischen

\footnotetext{
${ }^{31}$ Beide Filme beginnen mit der Präsentation eines Buches, das Rachels „Mission“ feiert, im US-amerikanischen Film ist sogar ihre Tochter die (befangene) Verfasserin.

32 Nach volkstümlichem jüdischen Glauben ein böser Geist, der in den Körper eines Lebenden eintritt und irrationales Verhalten und/oder Krankheiten hervorruft, vgl. Scholem (2007: 643644).
} 
Exorzismus zu vollziehen, bei dem neun Beteiligte sterben. Nachdem Casey zunächst gerettet scheint, erfährt sie jedoch am Ende des Films, dass sie mit Zwillingen schwanger ist - was ahnen lässt, dass die Heimsuchungen durch den Geist mit der nächsten Generation in eine neue Phase eintreten werden, das Böse also - genregerecht - nur vorläufig gebannt ist.

Mengele selbst tritt im Film lediglich in einer Rückblende auf, die ihn in Auschwitz mit internierten Kindern und bei Experimenten an Zwillingen zeigt. Caseys Großmutter charakterisiert ihn als "very handsome. Very polite“. Tatsächlich ist zu sehen, wie er den Kindern Bonbons schenkt und freundlich mit ihnen scherzt. Dieser Eindruck wird konterkariert durch die Darstellung von Mengeles Rücksichtslosigkeit während der Experimente; geradezu physisches Unbehagen erzeugt eine Szene, bei der er eine Injektionsspritze langsam an das Auge eines Kindes heranführt. Mengeles Interessen charakterisiert Caseys Großmutter als „obsessions“, sie schreibt ihm also eine mehr als nur sachliche Motivation seiner ,Forschung' zu: "[he] was obsessed with twins"; "one of [his] obsessions was eye colour". Beide Phänomene, die hier als Mengeles Interessensgebiete beschrieben werden - Zwillinge und die Verwandlung brauner in blaue Augen -, tauchen im Film ohne direkten Zusammenhang mit der Figur Mengeles wieder auf. In Caseys Familie werden beispielsweise überdurchschnittlich häufig Zwillinge geboren, und Caseys Augen verfärben sich zu Beginn des Films von braun zu blau, was ein Arzt als Effekt eines genetischen Mosaizismus diagnostiziert. Und wenn der Dybbuk Besitz von einem Menschen ergreift, zeigt sich dies äußerlich durch einen Wechsel der Augenfarbe zu einem unnatürlich wirkenden Blau. Historisch und im Film mit Mengele verbundene Thematiken werden auf diese Weise zu unheimlichen Versatzstücken in einem Horror-Plot. Indem der Film keine nachvollziehbare Erklärung für diese Phänomene anbietet, sie zugleich aber in der Rückblende mit Mengele assoziiert, entsteht der Eindruck eines rätselhaften Fortwirkens seiner Taten.

Dabei ist Mengele im Film das ,kleinere Bösé. Es sind zwar seine brutalen Experimente, die den Dybbuk in die Welt und in Caseys Familie bringen, die eigentliche Bedrohung ist jedoch der übernatürliche und - überraschenderweise - jüdische Geist, der auch nur durch ein als jüdisch markiertes Ritual ausgetrieben werden kann:33 "The Unborn is [...] a site of striking ideological confusion apparently suggesting Jewish complicity (albeit helpless or unconscious) in both the Holocaust itself and/or its contemporary resonances." (Langford 2013: 120) Der Film kriminalisiert so die Opfer, zumal angedeutet wird, dass Caseys Großmutter zur Mörderin ihres - wenn auch vom Dybbuk besessenen - Bruders wird. Anstatt, wie gemeinhin unumstritten, die

\footnotetext{
${ }_{33}$ Der Exorzismus findet unter der Leitung eines Rabbis in Anwesenheit von zehn Personen am Sabbat statt und besteht aus dem hebräischen Vorlesen einer (vermeintlichen) KabbalaPassage.
} 
Verbrechen in Auschwitz und die tödlichen Experimente an Kindern als extreme Gewalttaten darzustellen, erscheinen sie im Film lediglich als nicht genauer erklärter Auslöser "that empowers malevolent supernatural forces“ (Langford 2013: 120), denen sich dann die eigentliche Handlung widmet.

Der Rückgriff auf Mengele im Dienst einer genretypischen „Lust am filmischen Horror" (Vossen 2004: 9) ist ein problematisches Beispiel für „broader cultural ,uses' of the Holocaust" (Langford 2013: 121) „as a powerful reservoir of imaginary and dramatic impact" (Langford 2013: 120). Der Film nutzt die Figur des KZ-Arztes „as an atmospheric backdrop for his generic tale." (Dargis 2016) Die nationalsozialistischen Verbrechen werden instrumentalisiert und relativiert.

\section{FAZIT}

Die Figur des KZ-Arztes, im kollektiven Gedächtnis meist verkörpert durch Josef Mengele, wird in zahlreichen Spielfilmen fiktionalisiert, um verschiedene Bedürfnisse der Gegenwart zu erfüllen: Sie führt die Verbrechen des Holocaust vor Augen. Sie zeigt, wie Nazi-Verbrecher nach dem Krieg untertauchen und sich der Verfolgung entziehen konnten. Sie dient den nachfolgenden Generationen als Objekt der Auseinandersetzung. Und sie verselbständigt sich als Figur des ,Bösen' in diversen Genres. Während in den Holocaust-Filmen realistische Darstellungen dominieren, emanzipiert sich die Figur in den Filmen, deren Handlung nach 1945 spielt, von der Vorlage wirklicher Ereignisse. In den Filmen, die den Täter als Überlebenden inszenieren, werden an der Figur des KZ-Arztes zeitgenössische Probleme verhandelt: Medizinethik, Gentechnik, Sterbehilfe, McCarthy, der Vietnamkrieg sowie das Rechtssystem der Bundesrepublik Deutschland. Die Filme, die Nachfahren der Täter und Opfer in Szene setzen fragen nach dem richtigen Umgang mit dem Verbrecher; sie warnen sowohl vor einer zu großen Nähe als auch vor der Gefahr, in der Verfolgerrolle selbst zum Verbrecher zu werden. In Filmen des Horrorgenres wird der KZ-Arzt schließlich zu einer enthistorisierten Figur, die eine Sensationslust des Schreckens und eine Faszination der Macht und der Grausamkeit bedient. ${ }^{34}$

BIBLIOGRAPHIE

ADAMS, J. (2015), "Introduction“, in Representing Perpetrators in Holocaust Literature and Film, Adams, J. und Vice, S. (Hg.), London, Vallentine Mitchell, 1-24.

${ }^{34}$ Für Hinweise, Kommentare und gemeinsame Filmsichtungen danken wir Helmut Galle (São Paulo). 
Aly, G. (2013), Die Belasteten. ,Euthanasie' 1939-1945. Eine Gesellschaftsgeschichte, Frankfurt am Main, S. Fischer.

ANDERSCH A. (1982 [1980]), Der Vater eines Mörders. Erzählung, Zürich, Diogenes. ARENDT, H. (1994 [1963]), Eichmann in Jerusalem. A Report on the Banality of Evil, New York, Penguin.

BACHMANN, I. (1978 [1966]), "Der Fall Franza“, in Werke. Dritter Band, Bachmann, I., herausgegeben von von Weidenbaum, I. und Münster, München, Piper, 339-482.

BANGERT, A. (2008), „Changing Narratives and Images of the Holocaust: Tim Blake Nelson's The Grey Zone (2001)“, New Cinemas: Journal of Contemporary Film 6 (1), 17-32.

BANGerT, A. (2014), The Nazi Past in Contemporary German Film: Viewing Experiences of Intimacy and Immersion, Rochester, Camden House.

Baron, L. (2005), "The Grey Zone: The Cinema of Choiceless Choices", in Gray Zones: Ambiguity and Compromise in the Holocaust and its Aftermath, Petropolous, J. und Roth, J.K. (Hg.), Oxford and New York, Berghahn, 286292.

BENZENHÖFER, U. (2000), „Geschichte im Recht: NS-,Euthanasie' in neueren Gerichtsbeschlüssen zum Thema ,Behandlungsbegrenzung' (AG Hanau 1995, OLG Frankfurt 1998)“, in "Euthanasie' und die aktuelle SterbehilfeDebatte: die historischen Hintergründe medizinischer Ethik, Frewer, A. und Eickhoff, C. (Hg.), Frankfurt am Main und New York, Campus, 356-369.

Boozer, J. S. (1980), "Children of Hippocrates: Doctors in Nazi Germany“, The Annals of the American Academy of Political and Social Science 450, 83-97.

Boswell, M. (2012), Holocaust Impiety in Literature, Popular Music and Film, Basingstoke, Palgrave Macmillan UK.

Browning, C. R. (1992), Ordinary men. Reserve Police Battalion 101 and the Final Solution in Poland, New York, Haper Collins.

Burleigh, M. (2000), „Die Nazi-Analogie und die Debatten uber Euthanasie“ in ,Euthanasie‘ und die aktuelle Sterbehilfe-Debatte: die historischen Hintergründe medizinischer Ethik, Frewer, A. und Eickhoff, C. (Hg.), Frankfurt am Main und New York, Campus, 408-423.

DARGIS, M. (2009), "Mengele, the Holocaust and Horror Movie Staples“, in: The New York Times, 8.1.2009, online verfügbar [abgerufen am 29. September 2016]: <www.nytimes.com/2009/01/09/movies/09unbo.html>.

DICKS, H. V. (1972), Licensed Mass Murder, London, Chatto Heinemann und Sussex University Press.

FENELON, F. (1976), Sursis pour l'orchestre, Paris, Stock.

FREIMÜLLER, T. (2003), „Mediziner: Operation Volkskörper“ in Hitlers Eliten nach 1945, Frei, N. (Hg.), München, dtv, 13-65.

FREUD, S. (1999 [1919]), „Das Unheimliche“ in Gesammelte Werke. Chronologisch geordnet, Band XII, Freud, S., Frankfurt am Main, Fischer, 227-278.

Goldman, W. (1974), Marathon Man, New York, Delacorte. 
GoMEL, E. (2000), „From Dr. Moreau to Dr. Mengele: The Biological Sublime“, Poetics Today 21 (2), 393-421.

GRIMM, C. und HiLlEBRAND, I. (2009), Sterbehilfe. Rechtliche und ethische Aspekte (Ethik in den Biowissenschaften 8 - Sachstandsberichte des DRZE), Freiburg im Breisgau, Alber.

Gross, S. W. (1980), „Guilt and Innocence in Marathon Man“, Literature Film Quarterly 8 (1), 52-68.

HAKe, S. (2012), Screen Nazis. Cinema, History, and Democracy, Madison/London, The University of Wisconsin Press.

Henry, P. (2009), „,The Gray Zone“, Philosophy and Literature 33 (1), 150-166.

HochHuth, R. (1963), Der Stellvertreter. Ein christliches Trauerspiel, Reinbek, Rowohlt.

HofFMANN, E.T.A. (2000 [1816]), Der Sandmann, Frankfurt am Main, Insel.

Hohendorf, G. und Magull-Seltenreich, A. (1990) (Hg.), Von der Heilkunde zur Massentötung: Medizin im Nationalsozialismus, Heidelberg, Wunderhorn.

INSDORF, A. (2002), Inedible Shadows. Film and the Holocaust, Third Edition, Cambridge und New York, Cambridge University Press.

JÄGER, H. (1982 [1967]), Verbrechen unter totalitärer Herrschaft. Studien zur nationalsozialistischen Gewaltkriminalitat, Frankfurt am Main, Suhrkamp.

JüDIsCHes Museum BERLIN (Hg.) (2009), Tödliche Medizin: Rassenwahn im Nationalsozialismus, Göttingen, Wallstein.

Keller, S. (2003), Günzburg und der Fall Josef Mengele: Die Heimatstadt und die Jagd nach dem NS-Verbrecher, München, Oldenbourg.

KenEAlly, T. (1982), Schindler's Ark, London, Hodder \& Stoughton.

KERNER, A. (2011), Film and the Holocaust. New Perspectives on Dramas, Documentaries, and Experimental Films, New York und London, Continuum International Publishing Group.

KLEE, E. (1986), Was sie taten - Was sie wurden: Ärzte, Juristen und andere Beteiligte am Kranken- oder Judenmord, Frankfurt, S. Fischer.

KLEE, E. (1997), Auschwitz, die NS-Medizin und ihre Opfer, Frankfurt, S. Fischer.

KLEE, E. (2001), Deutsche Medizin im Dritten Reich: Karrieren vor und nach 1945, Frankfurt, S. Fischer.

KleEblatT, N. L. (Hg.) (2002), Mirroring Evil. Nazi Imagery / Recent Art, New Brunswick, Rutgers University Press.

KÖHNE, J. B. (2013), „Wissenschaft und Fiktion. Reproduktionsmedizin, menschliches Klonen und Ethik im Science-Fiction-Film The Boys from Brazil (1978)“" Österreichische Zeitschrift für Geschichtswissenschaften 24 (3) (Die Wahrheit des Film, herausgegeben von Ballhausen, T. und Barberi, A.), 55-78.

LANGFORD, B. (2013), „Globalizing the Holocaust. Fantasies of Annihilation in Contemporary Media Culture" in Holocaust Intersections. Genocide and Visual Culture at the New Millenium (Moving Image 4), Bangert, A., Gordon, 
R.S.C. und Saxton, L. (Hg.), London, LEGENDA/Modern Humanities Research Association and Maney Publishing, 112-129.

LEVI, P. (1993 [1986]), "Die Grauzone“ in Die Untergegangenen und die Geretteten, Levi, P., aus dem Italienischen von Kahn, M., München, dtv, 33-69.

LEVIN, I. (1976), The Boys from Brazil, New York, Random House.

Lifton, J. R. (1986), The Nazi Doctors: Medical Killing and the Psychology of Genocide, New York, Basic Books.

LoEWY, H. (2004), "Are we going to do this again? Nürnberg, Jerusalem, Frankfurt: Auschwitz und das Courtroom Drama" in Rechenschaften. Juristischer und literarischer Diskurs in der Auseinandersetzung mit den NSMassenverbrechen, Braese, S. (Hg.), Göttingen, Wallstein, 87-101.

Magilow D. H. (2012), „Introduction. Nazisploitation! The Nazi Image in LowBrow Cinema and Culture" in Nazisploitation! The Nazi Image in Low-Brow Cinema and Culture, Magilow, D.H., Bridges, E. und Vander Lugt K.T. (Hg.) New York und London, continuum, 1-18.

MAREK, R. (2012), „Der ,Klon' und seine Bilder - Über Faszination und Ästhetik in der Begriffsgeschichte", Forum interdisziplinäre Begriffsgeschichte, 1 (2), 16-43.

MAtTHÄUS, J. (1996), „What about the ,Ordinary Men’? The German Order Police and the Holocaust in the occupied Soviet union", Holocaust and Genocide Studies 10, 135-150.

Mauelshagen, C. (1995), Der Schatten des Vaters. Deutschsprachige Väterliteratur der siebziger und achtziger Jahre, (= Marburger germanistische Studien), Frankfurt am Main u.a., Peter Lang.

McGlothlin, E. (2010), „Theorizing the Perpetrator in Bernhard Schlink's The Reader and Martin Amis's Time's Arrow" in After Representation? The Holocaust, Literature, and Culture, Spargo, R.C. und Robert Ehrenreich, R. (Hg.), New Brunswick, NJ, Rutgers University Pess, 210-230.

McGothlin, E. (2015), ,", The Doctor Is Different‘. Ambivalent Ethics, Cinematic Heroics and the Figure of the Jewish Doctor in Tim Blake Nelson's The Grey Zone", in Holocaust Cinema in the Twenty-First Century, Kobrynski, O. und Bayer, G. (Hg.), New York und Chichester, Wallflower Press, 183-202.

MÜLLER, D. (2008), „Fritz Haarmann war der Vampir von Hannover” in Welt, 1.10.2008, online verfügbar [zugegriffen am 30. April 2018]: $<$ www.welt.de/vermischtes/article1994089/Fritz-Haarmann-war-derVampir-von-Hannover.html>.

NYISZLI, M. (2001 [1946]), Auschwitz. A Doctor's Eyewitness Account, übersetzt von Kremer, T. und Seaver, R., mit einem Vorwort von Bettelheim, B., New York, Arcade Publishing.

PAUL, G. (2002), „Von Psychopathen, Technokraten des Terrors und ,ganz gewöhnlichen' Deutschen. Die Täter der Shoah im Spiegel der Forschung" in Die Täter der Shoah. Fanatische Nationalsozialisten oder ganz normale Deutsche?, Paul, G. (Hg.), Göttingen, Wallstein Verlag, 13-90. 
PERL, G. (1948), I Was a Doctor in Auschwitz, New York, International Universities Press.

PerPer, J. A. und CinA, S. J. (2010), When Doctors Kill. Who, Why, and How, New York, Copernicus Books.

POSNER, G. L. und WARE, J. (2000), Mengele: The Complete Story, New York, Cooper Square Press.

Proctor, R. N. (2000), „Nazi-Ärzte, Rassenmedizin und ,lebensunwertes Leben” - von der Ideologie zur Euthanasie", in "Euthanasie' und die aktuelle Sterbehilfe-Debatte: die historischen Hintergründe medizinischer Ethik, Frewer, A. und Eickhoff, C. (Hg.), Frankfurt am Main/New York, Campus, 65-89.

PuenZO, L. (2011), Wakolda, Buenos Aires, Planeta.

Pugliese, S. G. (2003), „The Grey Zone“ [review], American Historical Review, 108 (3), 958-959.

REIDY, J. (2012), Vergessen, was Eltern sind. Relektüre und literaturgeschichtliche Neusituierung der angeblichen Väterliteratur, Göttingen, V \& R Unipress.

REIDY, J. (2015): "Väterliteratur" in Lexikon der "Vergangenheitsbewältigung" in Deutschland. Debatten- und Diskursgeschichte des Nationalsozialismus nach 1945, überarbeitete 3. Auflage, Fischer, T. und Lorenz, M.N., Bielefeld, transcript, 203-205.

Roelke, V., Hohendorf, G. und Rotzoll, M. (2000): „Psychiatrische Forschung, ,Euthanasie‘ und der ,Neue Mensch': Zur Debatte um Menschenbild und Wertsetzungen im Nationalsozialismus" in "Euthanasie' und die aktuelle Sterbehilfe-Debatte: die historischen Hintergründe medizinischer Ethik, Frewer, A. und Eickhoff, C. (Hg.), Frankfurt am Main und New York, Campus, 193-217.

SCHMUHL, H.-W. (2000), „Nationalsozialismus als Argument im aktuellen Medizinethik-Diskurs. Eine Zwischenbilanz" in ,Euthanasie' und die aktuelle Sterbehilfe-Debatte: die historischen Hintergründe medizinischer Ethik, Frewer, A. und Eickhoff, C. (Hg.), Frankfurt am Main und New York, Campus, 385-407.

SCHNEIDER, P. (1987), Vati, Darmstadt, Luchterhand.

ScHOlEM G. (2007), „Dibbuk (Dybbuk)“, in Encyclopaedia Judaica, 5, 2. Auflage, Detroit und New York, Macmillan, 643-644.

SHelley, M. (2012 [1818]): Frankenstein. The 1818 Text, Contexts, Criticism. A Norton Critical Edition, Hunter, J.P, (Hg.), New York und London, Norton \& Company.

SonTAG, S. (1991 [1971]), "Fascinating Fascism“, in Under the Sign of Saturn, Sontag, S., New York, Anchor, 71-105.

STEINHAUER, F. (2017), Der Werwolf von Hannover - Fritz Haarmann. Biografischer Kriminalroman, Meßkirch, Gmeiner.

STEINLE, M. (2013), „,Nous avons tous souffert': l'usage des signes de la Shoah dans les docufictions allemands contemporains" in La Shoah. Théâtre et 
cinéma aux limites de la représentation, Kleinberger, A. und Mesnard, P. (Hg.), Paris, Éditions Kimé.156-174.

StILES, A. (2009), „Literature in Mind: H. G. Wells and the Evolution of the Mad Scientist", Journal of the History of Ideas, 70 (2), 317-339.

UKLÁNSKI, P. (2015), Fatal Attraction, Brooks, R. (Hg.), New York, Rizzoli.

VÖLKLEIN, U. (1999), Josef Mengele. Der Arzt von Auschwitz, Göttingen, Steidl.

Vossen, U. (2004). „Einleitung“ in Filmgenres: Horrorfilm, Vossen, U. (Hg.), Stuttgart, Reclam, 9-27.

Wells, H. G. (2005 [1896]), The Island of Doctor Moreau, Parrinder, P. (Hg.), London, Penguin Classics.

WestermanN, E. B. (1998), „,'Ordinary Men' or ,ldeological Soldiers'? Police Battalion 310 in Russia, 1942", German Studies Review, 21, 41-68.

WulfF, H. J. (2002), "Zwischen Phantasie und Diskurs: Motive als Topoi in den Spielfilmen und journalistischen Texten der Gentechnik" in Humane Stammzellen: Therapeutische Optionen, ökonomische Perspektiven, mediale Vermittlung, Hauskeller, C. (Hg.), Lengerich, Pabst, 203-219.

ZIMMERMANN-ACKLIN, M. (2000), „,Der Schrecken nutzt sich ab’ - Zur Wechselwirkung von Geschichte und Ethik in der gegenwärtigen Euthanasie-Diskussion" in ,Euthanasie' und die aktuelle Sterbehilfe-Debatte: die historischen Hintergründe medizinischer Ethik, Frewer, A. und Eickhoff, C. (Hg.), Frankfurt am Main/New York, Campus, 448-470.

ZOFKA, Z. (1986), „Der KZ-Arzt Josef Mengele. Zur Typologie eines NSVerbrechers", Vierteljahrshefte für Zeitgeschichte, 34 (2), 245-267.

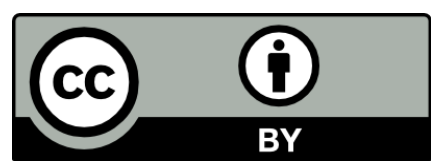

Los textos publicados en esta revista están sujetos - si no se indica lo contrario - a una licencia de Reconocimiento 3.0 España de Creative Commons. Puede compartirlos con terceros siempre que reconozca su autoría, la publicación inicial en esta revista y las condiciones de la licencia. 